\title{
TIME DOMAIN DECOMPOSITION IN FINAL VALUE OPTIMAL CONTROL OF THE MAXWELL SYSTEM*,**
}

\author{
John E. LAGNESE ${ }^{1}$ AND G. LEUGERING ${ }^{2}$
}

\begin{abstract}
We consider a boundary optimal control problem for the Maxwell system with a final value cost criterion. We introduce a time domain decomposition procedure for the corresponding optimality system which leads to a sequence of uncoupled optimality systems of local-in-time optimal control problems. In the limit full recovery of the coupling conditions is achieved, and, hence, the local solutions and controls converge to the global ones. The process is inherently parallel and is suitable for real-time control applications.
\end{abstract}

Mathematics Subject Classification. 65N55, 49M27, 35Q60.

Received December 17, 2001. Revised February 16, 2002.

\section{INTRODUCTION}

Problems of optimal control of electromagnetic waves arise in a variety of applications, e.g. in stealth technology, design and control of antennas, diffraction optics, magnetotellurics and related fields. In many important cases the objective to be met relates to the final values of both the electric and the magnetic fields involved. Hence, the problem of exact controllability has attracted considerable interest. See Lagnese [9] as an early paper and Phung [15] or Belishev and Glasman [2] for more recent ones on this topic. The list of references is far from being exhaustive. As a more realistic requirement, one typically asks for controls that bring the final states close to a given target configuration, that is, one wants to achieve optimal controls. While the papers mentioned focus on the case of constant permeabilities and permittivities, modern applications require dealing with heterogeneous materials. In addition, in most cases real-time requirements are to be met. Therefore, in order to obtain adjoint-based gradients and sensitivities in real-time, the large scale (or global) heterogeneous problem has to be reduced to smaller, more standard ones that can be processed in parallel. Domain decomposition of the Maxwell system for the purpose of simulation has been considered in e.g. Alonso and Valli [1] and Santos [16]. Domain decompositions with respect to optimality systems have been investigated by Lagnese [8]. In this paper we approach the problem of time domain decompositions of the optimality systems. Time decomposition methods (TDDM) appear to be a promising tool in real-time applications, as the sometimes long time horizon, which would be prohibitive in terms of numerical calculations, can be split into smaller time

Keywords and phrases: Maxwell system, optimal control, domain decomposition.

* Research of J.E.L. supported by the National Science Foundation through grant DMS-9972034.

** Research of G.L. supported by DFG grants Le595/12-2 and Le595/13-1

1 Department of Mathematics, Georgetown University, Washington, DC 20057, USA; e-mail: lagnese@math.georgetown.edu

2 Fachbereich Mathematik, Technische Universität Darmstadt, Schlossgartenstrasse 7, 64289 Darmstadt, Germany;

e-mail: leugering@mathematik.tu-darmstadt.de 
intervals, even down to the underlying time grid. Thus the problem can be recast into the framework of receding horizon control problems, or into what has come to be known as instantaneous control problems. An early reference to the possible utility of TDDM for the wave equation, but with no analysis provided, may be found in Benamou [3]. The transmission conditions suggested by Benamou contain a Robin-type condition in time which can be viewed as an approximation to transparent transmission conditions studied by Gander et al. [6]. Nevertheless, TDD-methods have been analyzed in the literature only very recently; see Heinkenschloss [7] and J.-L. Lions [13]. The common approach is to perform some sort of shooting method. That is to say, at the break points one restarts the dynamics with initial values that have to be optimized in order to achieve the correct continuity with respect to the time variable at the break points. While Heinkenschloss formulates the problem as an equality constrained optimal control problem with a Lagrangian relaxation, which is solved by a Gauss-Seidel type preconditioning of a GMRES-solve for a Shur-complement-type equation, Lions penalizes the defect of continuity across the break points. We, instead, mimic an augmented Lagrangian relaxation from spatial decompositions of elliptic problems, a strategy we have successfully used for wave equations in [10] (see also the references therein). This procedure leads to a sequence of local-in-time problems on the subintervals, which, in fact, turn out to be optimality systems for local-in-time optimal control problems. The procedure is completely parallel. We show convergence of the iteration and provide some useful a posteriori estimates of the error in the approximation. We emphasize that this novel time domain decomposition method can be combined with the corresponding spatial domain decomposition method such that the resulting iteration scheme provides a decomposition into space-time subdomains or, after discretization, even to space-time atoms on the finite element level.

\section{Setting the Problem}

Let $\Omega$ be a bounded, open, connected set in $\mathbb{R}^{3}$ with piecewise smooth, Lipschitz boundary $\Gamma$, and let $T>0$. We consider the Maxwell system

$$
\begin{aligned}
& \left\{\begin{array}{l}
\varepsilon E^{\prime}-\operatorname{rot} H+\sigma E=F \\
\mu H^{\prime}+\operatorname{rot} E=G
\end{array} \quad \text { in } Q:=\Omega \times(0, T)\right. \\
& H_{\tau}-\alpha(\nu \wedge E)=J \quad \text { on } \Sigma:=\Gamma \times(0, T) \\
& E(0)=\phi, \quad H(0)=\psi \quad \text { in } \Omega .
\end{aligned}
$$

Here $^{\prime}=\partial / \partial t, \wedge$ is the standard vector product operation, $\nu$ denotes the exterior pointing unit normal vector to $\Gamma, H_{\tau}$ is the tangential component of $H$, that is,

$$
H_{\tau}=H-(H \cdot \nu) \nu=\nu \wedge(H \wedge \nu),
$$

and $\alpha \in L^{\infty}(\Gamma), \alpha(x) \geq \alpha_{0}>0$. Further, $\varepsilon=\left(\varepsilon^{j k}(x)\right), \mu=\left(\mu^{j k}(x)\right)$ and $\sigma=\left(\sigma^{j k}(x)\right)$ are $3 \times 3$ Hermitian matrices with $L^{\infty}(\Omega)$ entries such that $\varepsilon$ and $\mu$ are uniformly positive definite and $\sigma \geq 0$ in $\Omega$. The functions $F$, $G \in L^{1}\left(0, T ; \mathcal{L}^{2}(\Omega)\right)$ are given while $J$ is a control input and is taken from the class

$$
\mathcal{U}=\mathcal{L}_{\tau}^{2}(\Sigma):=\left\{J \mid J \in L^{2}\left(0, T ; \mathcal{L}_{\tau}^{2}(\Gamma)\right)\right\}
$$

where $\mathcal{L}_{\tau}^{2}(\Gamma)$ denotes vector valued $\mathcal{L}^{2}(\Gamma)$ functions having a zero normal component.

When $J=0$ and $\alpha=1$, the boundary condition $(2.1)_{3}$ is known as the Silver-Müller boundary condition. It is the first approximation to the so-called transparent boundary condition, which corresponds to the transmission of electromagnetic waves through the boundary without reflections. In general, when $\alpha>0$ on a set on positive measure in $\Gamma$, the boundary condition $(2.1)_{3}$ is dissipative. That is, if one defines the electromagnetic energy by

$$
\mathcal{E}(t)=\int_{\Omega}(\varepsilon E \cdot E+\mu H \cdot H) \mathrm{d} x
$$


then, in the absence of external inputs $F, G, J$, the functional $\mathcal{E}(t)$ is nonincreasing. Moreover, the boundary condition $(2.1)_{3}$ is also regularizing. That is to say, if $\mathcal{E}(0)<\infty$, if $F, G$ and $J$ have regularity assumed above, and if the support of $J$ is contained in the support of $\alpha$, the solution of (2.1) satisfies $\mathcal{E}(t)<\infty$ for all $t \geq 0$. On the other hand, if $\alpha \equiv 0$ and $J \not \equiv 0$ then, in general, solutions of (2.1) will have less regularity.

In what follows, function spaces of $\mathcal{C}$-valued functions are denoted by capital Roman letters, while function spaces of $\mathcal{C}^{3}$-valued functions are denoted by capital script letters. We use $\alpha \cdot \beta$ to denote the natural scalar product in $\mathcal{C}^{3}$, i.e., $\alpha \cdot \beta=\sum_{j=1}^{3} \alpha_{j} \bar{\beta}_{j}$, and write $\langle\cdot, \cdot\rangle$ for the natural scalar product in various function spaces such as $L^{2}(\Omega)$ and $\mathcal{L}^{2}(\Omega)$. A subscript may sometimes be added to avoid confusion. The spaces $L^{2}(\Omega)$ and $\mathcal{L}^{2}(\Omega)$ denote the usual spaces of Lebesque square integrable $\mathcal{C}$-valued functions and $\mathcal{C}^{3}$-valued functions, respectively, and, similarly, for the Sobolev spaces $H^{s}(\Omega), \mathcal{H}^{s}(\Omega)$. We also denote by $\mathcal{L}_{\varepsilon}^{2}(\Omega)$ the space $\mathcal{L}^{2}(\Omega)$ with weight matrix $\varepsilon$ and $\langle\phi, \psi\rangle_{\varepsilon}:=\langle\varepsilon \phi, \psi\rangle$ the scalar product of $\phi$ and $\psi$ in that space. With this notation, the energy space $\mathcal{H}:=\mathcal{L}_{\varepsilon}^{2}(\Omega) \times \mathcal{L}_{\mu}^{2}(\Omega)$.

When $(\phi, \psi) \in \mathcal{H},(F, G) \in L^{1}(0, T ; \mathcal{H})$ and $J \in \mathcal{L}_{\tau}^{2}(\Sigma)$, it may be proved that the system (2.1) has a unique solution with regularity $(E, H) \in C([0, T] ; \mathcal{H}),\left.\nu \wedge E\right|_{\Sigma} \in \mathcal{L}_{\tau}^{2}(\Sigma)$ and, moreover, the linear map from the data to $\left((E, H),\left.\nu \wedge E\right|_{\Sigma}\right)$ is continuous in the indicated spaces (see, e.g. [11]). Therefore, given $\left(E_{T}, H_{T}\right) \in \mathcal{H}$, we may consider the final value optimal control problem

$$
\inf _{J \in \mathcal{U}} \mathcal{J}(J), \quad \mathcal{U}:=\mathcal{L}_{\tau}^{2}(\Sigma)
$$

subject to $(2.1)$, where

$$
\mathcal{J}(J)=\frac{1}{2} \int_{\Sigma}|J|^{2} \mathrm{~d} \Sigma+\frac{z}{2}\left\|(E(T), H(T))-\left(E_{T}, H_{T}\right)\right\|_{\mathcal{H}}^{2}
$$

with $z$ a positive penalty parameter. Since the cost functional $\mathcal{J}$ is convex and in view of the properties of the map $((\phi, \psi),(F, G), J) \mapsto(E, H)$, it is standard theory that there exists a unique optimal control $J_{\text {opt }}$. It is shown in the next section that $J_{\mathrm{opt}}$ is given by

$$
J_{\text {opt }}=\left.\nu \wedge P\right|_{\Sigma}
$$

where $(P, Q)$ is the solution of the backwards running adjoint system

$$
\begin{gathered}
\begin{cases}\varepsilon P^{\prime}-\operatorname{rot} Q-\sigma P=0 & \text { in } Q \\
\mu Q^{\prime}+\operatorname{rot} P=0 & \text { on } \Sigma\end{cases} \\
Q_{\tau}+\alpha(\nu \wedge P)=0 \\
\begin{cases}P(T)=z\left(E(T)-E_{T}\right) & \text { in } \Omega . \\
Q(T)=z\left(H(T)-H_{T}\right) & \end{cases}
\end{gathered}
$$

The purpose of this paper is to develop a convergent time domain decomposition method (TDDM) to approximate the solution of the optimality system $(2.1,2.4,2.5)$, and to derive certain a posteriori estimate of the error in the approximation.

Our TDDM is introduced in Section 4, and it is shown that each of the local problems entering into the algorithm is itself an optimality system. Convergence of the algorithm is established in Section 5. A posteriori estimates of the error in the approximation in terms of the mismatch of the iterates at the break points are derived in Section 6.

Remark 2.1. Instead of $\mathcal{L}_{\tau}^{2}(\Sigma)$ one may choose as the control space $\mathcal{U}$ those $\mathcal{L}_{\tau}^{2}(\Sigma)$ functions that are supported in $\widetilde{\Gamma} \times(0, T)$, where $\widetilde{\Gamma}$ is a subset of $\Gamma$ of positive 2 -dimensional measure. The analysis presented below may easily be adapted to this more general setting with only minor modifications. 


\section{The optimality SYSTEM}

The necessary and sufficient condition for optimality is that the directional derivative of $\mathcal{J}$ at $J_{\mathrm{opt}}$ in the direction of $\hat{J}$ is equal to zero. Therefore $J_{\mathrm{opt}}$ is the solution of the variational equation

$$
\int_{\Sigma} J_{\mathrm{opt}} \cdot \hat{J} \mathrm{~d} \Sigma+z\left\langle\left(E(T)-E_{T}, H(T)-H_{T}\right),(\hat{E}(T), \hat{H}(T))\right\rangle_{\mathcal{H}}=0, \quad \forall \hat{J} \in \mathcal{L}_{\tau}^{2}(\Sigma),
$$

where $(\hat{E}, \hat{H})$ is the solution of

$$
\begin{aligned}
& \left\{\begin{array}{l}
\varepsilon \hat{E}^{\prime}-\operatorname{rot} \hat{H}+\sigma \hat{E}=0 \\
\mu \hat{H}^{\prime}+\operatorname{rot} \hat{E}=0
\end{array} \quad \text { in } Q\right. \\
& \hat{H}_{\tau}-\alpha(\nu \wedge \hat{E})=\hat{J} \quad \text { on } \Sigma \\
& \hat{E}(0)=\hat{H}(0)=0 \quad \text { in } \Omega \text {. }
\end{aligned}
$$

Let $(P, Q)$ be the solution of $(2.5)$. Then $(P, Q) \in C([0, T] ; \mathcal{H})$ and $\left.\nu \wedge P\right|_{\Sigma} \in \mathcal{U}$. We have

$$
0=\int_{0}^{T}\left\{\left\langle\varepsilon P^{\prime}-\operatorname{rot} Q-\sigma P, \hat{E}\right\rangle+\left\langle\mu Q^{\prime}+\operatorname{rot} P, \hat{H}\right\rangle\right\} \mathrm{d} t .
$$

By utilizing Green's formula

$$
\begin{aligned}
\langle\operatorname{rot} \phi, \psi\rangle & =\langle\phi, \operatorname{rot} \psi\rangle+\int_{\Gamma}(\nu \wedge \phi) \cdot \psi_{\tau} \mathrm{d} \Gamma \\
& =\langle\phi, \operatorname{rot} \psi\rangle-\int_{\Gamma} \phi_{\tau} \cdot(\nu \wedge \psi) \mathrm{d} \Gamma
\end{aligned}
$$

we may rewrite (3.2) as

$$
0=z\left\langle\left(E(T)-E_{T}, H(T)-H_{T}\right),(\hat{E}(T), \hat{H}(T))\right\rangle_{\mathcal{H}}+\int_{\Sigma}(\nu \wedge P) \cdot \hat{J} \mathrm{~d} \Sigma, \quad \forall \hat{J} \in \mathcal{L}_{\tau}^{2}(\Sigma) .
$$

Thus (2.4) follows from (3.1) and (3.4).

\section{Time DOMAIN DECOMPOSITION}

We introduce a partition of the time interval $[0, T]$ by setting

$$
0=T_{0}<T_{1}<\cdots<T_{K}<T_{K+1}=T
$$

and thereby decompose $[0, T]$ into $K+1$ subintervals $I_{k}:=\left[T_{k}, T_{k+1}\right], k=0, \ldots, K$. We further introduce locally defined functions $E_{k}=\left.E\right|_{I_{k}}, H_{k}=\left.H\right|_{I_{k}}$, and so forth. We proceed to decompose the optimality system $(2.1,2.4,2.5)$ into the following local systems defined on $I_{k}, k=0, \ldots, K$ :

$$
\begin{array}{cl} 
\begin{cases}\varepsilon E_{k}^{\prime}-\operatorname{rot} H_{k}+\sigma E_{k}=F_{k} & \\
\mu H_{k}^{\prime}+\operatorname{rot} E_{k}=G_{k} & \text { in } Q_{k}:=\Omega \times I_{k}\end{cases} \\
H_{k \tau}-\alpha\left(\nu \wedge E_{k}\right)=\nu \wedge P_{k} & \text { on } \Sigma_{k}:=\Gamma \times I_{k},
\end{array}
$$




$$
\begin{array}{cl} 
\begin{cases}\varepsilon P_{k}^{\prime}-\operatorname{rot} Q_{k}-\sigma P_{k}=0 & \\
\mu Q_{k}^{\prime}+\operatorname{rot} P_{k}=0 & \text { in } Q_{k}\end{cases} \\
Q_{k \tau}+\alpha\left(\nu \wedge P_{k}\right)=0 & \text { on } \Sigma_{k} .
\end{array}
$$

The "initial" values $\left(E_{k}\left(T_{k}\right), H_{k}\left(T_{k}\right)\right)$ and $\left(P_{k}\left(T_{k+1}\right), Q_{k}\left(T_{k+1}\right)\right)$ for $(4.24 .3)$ are given through the coupling conditions

$$
\begin{gathered}
E_{k}\left(T_{k}\right)=E_{k-1}\left(T_{k}\right), \quad H_{k}\left(T_{k}\right)=H_{k-1}\left(T_{k}\right), \quad k=1, \ldots, K \\
P_{k}\left(T_{k+1}\right)=P_{k+1}\left(T_{k+1}\right), \quad Q_{k}\left(T_{k+1}\right)=Q_{k+1}\left(T_{k+1}\right), \quad k=K-1, \ldots, 0
\end{gathered}
$$

together with

$$
\begin{aligned}
E_{0}(0)=\phi, & H_{0}(0)=\psi, \\
P_{K}(T)=z\left(E_{K}(T)-E_{T}\right), & Q_{K}(T)=z\left(H_{K}(T)-H_{T}\right) .
\end{aligned}
$$

We now uncouple the local problems by uncoupling (4.4) through an iteration as follows:

$$
\begin{gathered}
\left\{\begin{array}{l}
\beta E_{k}^{n+1}\left(T_{k+1}\right)-P_{k}^{n+1}\left(T_{k+1}\right)=\mu_{k, k+1}^{n} \\
\beta H_{k}^{n+1}\left(T_{k+1}\right)-Q_{k}^{n+1}\left(T_{k+1}\right)=\eta_{k, k+1}^{n}, \quad k=0, \ldots, K-1
\end{array}\right. \\
\left\{\begin{array}{l}
\beta E_{k}^{n+1}\left(T_{k}\right)+P_{k}^{n+1}\left(T_{k}\right)=\mu_{k, k-1}^{n} \\
-\beta H_{k}^{n+1}\left(T_{k}\right)-Q_{k}^{n+1}\left(T_{k}\right)=\eta_{k, k-1}^{n}, \quad k=1, \ldots, K,
\end{array}\right.
\end{gathered}
$$

where $\beta>0$ and

$$
\begin{gathered}
\mu_{k, k+1}^{n}=\beta E_{k+1}^{n}\left(T_{k+1}\right)-P_{k+1}^{n}\left(T_{k+1}\right) \\
\eta_{k, k+1}^{n}=\beta H_{k+1}^{n}\left(T_{k+1}\right)-Q_{k+1}^{n}\left(T_{k+1}\right) \\
\mu_{k, k-1}^{n}=\beta E_{k-1}^{n}\left(T_{k}\right)+P_{k-1}^{n}\left(T_{k}\right) \\
\eta_{k, k-1}^{n}=-\beta H_{k-1}^{n}\left(T_{k}\right)-Q_{k-1}^{n}\left(T_{k}\right) .
\end{gathered}
$$

It is readily seen that in the limit of $(4.6,4.7)$ one recovers $(4.4)$, so that $(4.6,4.7)$ are consistent with (4.4).

For the convenience of the reader we write down the complete set of uncoupled systems:

$$
\begin{gathered}
\left\{\begin{array}{l}
\varepsilon\left(E_{k}^{n+1}\right)^{\prime}-\operatorname{rot} H_{k}^{n+1}+\sigma E_{k}^{n+1}=F_{k} \\
\mu\left(H_{k}^{n+1}\right)^{\prime}+\operatorname{rot} E_{k}^{n+1}=G_{k}
\end{array}\right. \\
\left\{\begin{array}{l}
\varepsilon\left(P_{k}^{n+1}\right)^{\prime}-\operatorname{rot} Q_{k}^{n+1}-\sigma P_{k}^{n+1}=0 \\
\mu\left(Q_{k}^{n+1}\right)^{\prime}+\operatorname{rot} P_{k}^{n+1}=0
\end{array}\right. \\
\left\{\begin{array}{l}
H_{k \tau}^{n+1}-\alpha\left(\nu \wedge E_{k}^{n+1}\right)=\nu \wedge P_{k}^{n+1} \\
Q_{k \tau}^{n+1}+\alpha\left(\nu \wedge P_{k}^{n+1}\right)=0
\end{array}\right. \\
\left\{\begin{array}{l}
E_{K}^{n+1}(T)=z\left(E_{K}^{n+1}(T)-E_{T}\right) \\
Q_{K}^{n+1}(T)=z\left(H_{K}^{n+1}(T)-H_{T}\right)
\end{array}\right. \\
\end{gathered}
$$

subject to $(4.6,4.7)$. 
We wish to show that for $\left(\mu_{k, k+1}^{n}, \eta_{k, k+1}^{n}\right)$ and $\left(\mu_{k, k-1}^{n}, \eta_{k, k-1}^{n}\right)$ given in $\mathcal{H}$, the local problems are well posed for $k=0, \ldots, K$. To do so we shall show that the local problem with index $k$ is in fact an optimality system concentrated on the interval $I_{k}, k=0, \ldots, K$.

Proposition 4.1. Assume that $\left(\mu_{k, k+1}^{n}, \eta_{k, k+1}^{n}\right) \in \mathcal{H},\left(\mu_{k, k-1}^{n}, \eta_{k, k-1}^{n}\right) \in \mathcal{H}$. Set

$$
\begin{aligned}
\mathcal{J}_{k}\left(J_{k}, h_{k, k-1}, g_{k, k-1}\right)= & \frac{1}{2} \int_{\Sigma_{k}}\left|J_{k}\right|^{2} \mathrm{~d} \Sigma+\frac{1}{2 \beta}\left\{\left\|\beta E_{k}\left(T_{k+1}\right)-\mu_{k, k+1}^{n}\right\|_{\varepsilon}^{2}\right. \\
& \left.+\left\|\beta H_{k}\left(T_{k+1}\right)-\eta_{k, k+1}^{n}\right\|_{\mu}^{2}+\left\|\left(h_{k, k-1}, g_{k, k-1}\right)\right\|_{\mathcal{H}}^{2}\right\}, \quad k=1, \ldots, K-1, \\
\mathcal{J}_{K}\left(J_{K}, h_{K, K-1}, g_{K, K-1}\right)= & \frac{1}{2} \int_{\Sigma_{K}}\left|J_{K}\right|^{2} \mathrm{~d} \Sigma \\
& +\frac{z}{2}\left\|\left(E_{K}(T), H_{K}(T)\right)-\left(E_{T}, H_{T}\right)\right\|_{\mathcal{H}}^{2}+\frac{1}{2 \beta}\left\|\left(h_{K, K-1}, g_{K, K-1}\right)\right\|_{\mathcal{H}}^{2}, \\
\mathcal{J}_{0}\left(J_{0}\right)= & \frac{1}{2} \int_{\Sigma_{0}}\left|J_{0}\right|^{2} \mathrm{~d} \Sigma+\frac{1}{2 \beta}\left\{\left\|\beta E_{0}\left(T_{1}\right)-\mu_{0,1}^{n}\right\|_{\varepsilon}^{2}+\left\|\beta H_{0}\left(T_{1}\right)-\eta_{0,1}^{n}\right\|_{\mu}^{2}\right\} .
\end{aligned}
$$

For $k=0 \ldots, K$, the system $(4.8-4.10,4.6)$ is the optimality system for the optimal control problem $\inf _{\mathcal{U}_{k}} \mathcal{J}_{k}$, where $\mathcal{U}_{k}=\mathcal{L}_{\tau}^{2}\left(\Sigma_{k}\right) \times \mathcal{H}$ for $k=1, \ldots, K, \mathcal{U}_{0}=\mathcal{L}_{\tau}^{2}\left(\Sigma_{0}\right)$, subject to

$$
\begin{aligned}
& \begin{cases}\varepsilon E_{k}^{\prime}-\operatorname{rot} H_{k}+\sigma E_{k}=F_{k} & \text { in } Q_{k} \\
\mu H_{k}^{\prime}+\operatorname{rot} E_{k}=G_{k} & \text { on } \Sigma_{k}\end{cases} \\
& H_{k \tau}-\alpha\left(\nu \wedge E_{k}\right)=J_{k} \quad
\end{aligned}
$$

and

$$
\begin{gathered}
\left\{\begin{array}{l}
E_{k}\left(T_{k}\right)=\frac{1}{\beta}\left(h_{k, k-1}+\mu_{k, k-1}^{n}\right) \\
H_{k}\left(T_{k}\right)=-\frac{1}{\beta}\left(g_{k, k-1}+\eta_{k, k-1}^{n}\right) \text { in } \Omega, \quad k=1, \ldots, K,
\end{array}\right. \\
E_{0}(0)=\phi, \quad H_{0}(0)=\psi \text { in } \Omega .
\end{gathered}
$$

Remark 4.1. In the local optimal control problem $\inf _{\mathcal{U}_{k}} \mathcal{J}_{k}\left(J_{k}, h_{k, k-1}, g_{k, k-1}\right)$, the control $J_{k}$ is, in the terminology of J.-L. Lions and O. Pironneau, the effective control and the controls $h_{k, k-1}, g_{k, k-1}$ are virtual, or artificial controls; see [12-14].

Proof. We give the proof only for $k=1, \ldots, K-1$ since the proofs in the two remaining cases are similar. The necessary and sufficient condition that $J_{k}, h_{k, k-1}, g_{k, k-1}$ be optimal is that the directional derivative of $\mathcal{J}_{k}$ at $J_{k}, h_{k, k-1}, g_{k, k-1}$ in the direction of $\hat{J}_{k}, \hat{h}_{k, k-1}, \hat{g}_{k, k-1}$ be zero. This leads to the variational equation

$$
\begin{aligned}
0= & \int_{\Sigma_{k}} J_{k} \cdot \hat{J}_{k} \mathrm{~d} \Sigma+\left\langle\beta E_{k}\left(T_{k+1}\right)-\mu_{k, k+1}^{n}, \hat{E}_{k}\left(T_{k+1}\right)\right\rangle_{\varepsilon} \\
& +\left\langle\beta H_{k}\left(T_{k+1}\right)-\eta_{k, k+1}^{n}, \hat{H}_{k}\left(T_{k+1}\right)\right\rangle_{\mu}+\frac{1}{\beta}\left\langle\left(h_{k, k-1}, g_{k, k-1}\right),\left(\hat{h}_{k, k-1}, \hat{g}_{k, k-1}\right)\right\rangle_{\mathcal{H}}, \\
& \forall\left(\hat{J}_{k}, \hat{h}_{k, k-1}, \hat{g}_{k, k-1}\right) \in \mathcal{U}_{k}
\end{aligned}
$$


where

$$
\begin{aligned}
& \begin{cases}\varepsilon \hat{E}_{k}^{\prime}-\operatorname{rot} \hat{H}_{k}+\sigma \hat{E}_{k}=0 & \\
\mu \hat{H}_{k}^{\prime}+\operatorname{rot} \hat{E}_{k}=0 & \text { in } Q_{k}\end{cases} \\
& \hat{H}_{k \tau}-\alpha\left(\nu \wedge \hat{E}_{k}\right)=\hat{J}_{k} \quad \text { on } \Sigma_{k} \\
& \hat{E}_{k}\left(T_{k}\right)=\frac{1}{\beta} \hat{h}_{k, k-1}, \quad \hat{H}_{k}\left(T_{k}\right)=-\frac{1}{\beta} \hat{g}_{k, k-1} \quad \text { in } \Omega \text {. }
\end{aligned}
$$

Introduce $\left(P_{k}, Q_{k}\right)$ as the solution of

$$
\begin{gathered}
\begin{cases}\varepsilon P_{k}^{\prime}-\operatorname{rot} Q_{k}-\sigma P_{k}=0 & \text { in } Q_{k} \\
\mu Q_{k}^{\prime}+\operatorname{rot} P_{k}=0 & \text { on } \Sigma_{k}\end{cases} \\
Q_{k \tau}+\alpha\left(\nu \wedge P_{k}\right)=0 \\
\begin{cases}P_{k}\left(T_{k+1}\right)=\beta E_{k}\left(T_{k+1}\right)-\mu_{k, k+1}^{n} \\
Q_{k}\left(T_{k+1}\right)=\beta H_{k}\left(T_{k+1}\right)-\eta_{k, k+1}^{n} & \text { in } \Omega .\end{cases}
\end{gathered}
$$

We have

$$
\begin{aligned}
0= & \int_{T_{k}}^{T_{k+1}}\left\{\left\langle\varepsilon P_{k}^{\prime}-\operatorname{rot} Q_{k}-\sigma P_{k}, \hat{E}_{k}\right\rangle+\left\langle\mu Q_{k}^{\prime}+\operatorname{rot} P_{k}, \hat{H}_{k}\right\rangle\right\} \mathrm{d} t \\
= & \left.\left\langle\left(P_{k}(t), Q_{k}(t)\right),\left(\hat{E}_{k}(t), \hat{H}_{k}(t)\right)\right\rangle_{\mathcal{H}}\right|_{t=T_{k}} ^{T_{k+1}} \\
& +\int_{\Sigma_{k}}\left[Q_{k \tau} \cdot\left(\nu \wedge \hat{E}_{k}\right)+\left(\nu \wedge P_{k}\right) \cdot \hat{H}_{k \tau}\right] \mathrm{d} \Sigma
\end{aligned}
$$

where we have used (3.3). By utilizing (4.18) and (4.19) it is seen that (4.20) may be written

$$
\begin{aligned}
0= & \left\langle\left(\beta E_{k}\left(T_{k+1}\right)-\mu_{k, k+1}^{n}, \beta H_{k}\left(T_{k+1}\right)-\eta_{k, k+1}^{n}\right),\left(\hat{E}_{k}\left(T_{k+1}\right), \hat{H}_{k}\left(T_{k+1}\right)\right)\right\rangle_{\mathcal{H}} \\
& -\left\langle\left(P_{k}\left(T_{k}\right), Q_{k}\left(T_{k}\right)\right),\left(\frac{1}{\beta} \hat{h}_{k, k-1},-\frac{1}{\beta} \hat{g}_{k, k-1}\right)\right\rangle_{\mathcal{H}}+\int_{\Sigma_{k}}\left(\nu \wedge P_{k}\right) \cdot \hat{J}_{k} \mathrm{~d} \Sigma .
\end{aligned}
$$

It now follows from (4.21) and (4.17) that

$$
J_{k}=\left.\nu \wedge P_{k}\right|_{\Sigma_{k}}, \quad h_{k, k-1}=-P_{k}\left(T_{k}\right), \quad g_{k, k-1}=Q_{k}\left(T_{k}\right)
$$

from which the conclusion of Proposition 4.1 follows immediately.

Corollary 4.1. Let $\left.\left(\mu_{k, k+1}^{0}, \eta_{k, k+1}^{0}\right)\right|_{k=0} ^{K-1}$ and $\left.\left(\mu_{k, k-1}^{0}, \eta_{k, k-1}^{0}\right)\right|_{k=1} ^{K}$ be given arbitrarily in $\mathcal{H}$. Then the iterative procedure described by (4.6-4.10) is well defined for $n=0,1, \ldots$

\section{Convergence of the iterates}

We consider the iterative procedure with the basic step given by (4.6-4.10). In fact, we shall consider a relaxation of the iteration step $(4.6,4.7)$. Thus we introduce a relaxation parameter $\epsilon \in[0,1)$ and consider the 
iterative step $(4.6,4.7)$ with under relaxation:

$$
\begin{aligned}
\beta E_{k}^{n+1}\left(T_{k+1}\right)-P_{k}^{n+1}\left(T_{k+1}\right) & =(1-\epsilon) \mu_{k, k+1}^{n}+\epsilon\left(\beta E_{k}^{n}\left(T_{k+1}\right)-P_{k}^{n}\left(T_{k+1}\right)\right) \\
\beta H_{k}^{n+1}\left(T_{k+1}\right)-Q_{k}^{n+1}\left(T_{k+1}\right) & =(1-\epsilon) \eta_{k, k+1}^{n}+\epsilon\left(\beta H_{k}^{n}\left(T_{k+1}\right)-Q_{k}^{n}\left(T_{k+1}\right)\right) \\
\beta E_{k}^{n+1}\left(T_{k}\right)+P_{k}^{n+1}\left(T_{k}\right) & =(1-\epsilon) \mu_{k, k-1}^{n}+\epsilon\left(\beta E_{k}^{n}\left(T_{k}\right)+P_{k}^{n}\left(T_{k}\right)\right) \\
-\beta H_{k}^{n+1}\left(T_{k}\right)-Q_{k}^{n+1}\left(T_{k}\right) & =(1-\epsilon) \eta_{k, k-1}^{n}+\epsilon\left(-\beta H_{k}^{n}\left(T_{k}\right)-Q_{k}^{n}\left(T_{k}\right)\right) .
\end{aligned}
$$

We introduce the errors

$$
\widetilde{E}_{k}^{n}=E_{k}^{n}-E_{k}, \quad \widetilde{H}_{k}^{n}=H_{k}^{n}-H_{k}, \quad \widetilde{P}_{k}^{n}=P_{k}^{n}-P_{k}, \quad \widetilde{Q}_{k}^{n}=Q_{k}^{n}-Q_{k} .
$$

These satisfy

$$
\begin{gathered}
\begin{cases}\varepsilon\left(\widetilde{E}_{k}^{n+1}\right)^{\prime}-\operatorname{rot} \widetilde{H}_{k}^{n+1}+\sigma \widetilde{E}_{k}^{n+1}=0 \\
\mu\left(\widetilde{H}_{k}^{n+1}\right)^{\prime}+\operatorname{rot} \widetilde{E}_{k}^{n+1}=0 & \text { in } Q_{k}\end{cases} \\
\begin{cases}\varepsilon\left(\widetilde{P}_{k}^{n+1}\right)^{\prime}-\operatorname{rot} \widetilde{Q}_{k}^{n+1}-\sigma \widetilde{P}_{k}^{n+1}=0 \\
\mu\left(\widetilde{Q}_{k}^{n+1}\right)^{\prime}+\operatorname{rot} \widetilde{P}_{k}^{n+1}=0\end{cases} \\
\left\{\begin{array}{l}
\widetilde{H}_{k \tau}^{n+1}-\alpha\left(\nu \wedge \widetilde{E}_{k}^{n+1}\right)=\nu \wedge \widetilde{P}_{k}^{n+1} \\
Q_{k \tau}^{n+1}+\alpha\left(\nu \wedge P_{k}^{n+1}\right)=0
\end{array}\right. \\
\widetilde{P}_{K}^{n+1}(T)=z \widetilde{E}_{0}^{n+1}(0)=\widetilde{H}_{K}^{n+1}(T), \quad \widetilde{Q}_{K}^{n+1}(0)=0 \\
\end{gathered}
$$

subject to

$$
\begin{aligned}
& \left\{\begin{array}{l}
\beta \widetilde{E}_{k}^{n+1}\left(T_{k+1}\right)-\widetilde{P}_{k}^{n+1}\left(T_{k+1}\right)=\tilde{\mu}_{k, k+1}^{n} \\
\beta \widetilde{H}_{k}^{n+1}\left(T_{k+1}\right)-\widetilde{Q}_{k}^{n+1}\left(T_{k+1}\right)=\tilde{\eta}_{k, k+1}^{n}, \quad k=0, \ldots, K-1 \\
\beta \widetilde{E}_{k}^{n+1}\left(T_{k}\right)+\widetilde{P}_{k}^{n+1}\left(T_{k}\right)=\tilde{\mu}_{k, k-1}^{n} \\
-\beta \widetilde{H}_{k}^{n+1}\left(T_{k}\right)-\widetilde{Q}_{k}^{n+1}\left(T_{k}\right)=\tilde{\eta}_{k, k-1}^{n}, \quad k=1, \ldots, K
\end{array}\right.
\end{aligned}
$$

where, for $n \geq 1$,

$$
\begin{gathered}
\tilde{\mu}_{k, k+1}^{n}=(1-\epsilon)\left(\beta \widetilde{E}_{k+1}^{n}\left(T_{k+1}\right)-\widetilde{P}_{k+1}^{n}\left(T_{k+1}\right)\right)+\epsilon\left(\beta \widetilde{E}_{k}^{n}\left(T_{k+1}\right)-\widetilde{P}_{k}^{n}\left(T_{k+1}\right)\right) \\
\tilde{\eta}_{k, k+1}^{n}=(1-\epsilon)\left(\beta \widetilde{H}_{k+1}^{n}\left(T_{k+1}\right)-\widetilde{Q}_{k+1}^{n}\left(T_{k+1}\right)\right)+\epsilon\left(\beta \widetilde{H}_{k}^{n}\left(T_{k+1}\right)-\widetilde{Q}_{k}^{n}\left(T_{k+1}\right)\right) \\
\tilde{\mu}_{k, k-1}^{n}=(1-\epsilon)\left(\beta \widetilde{E}_{k-1}^{n}\left(T_{k}\right)+\widetilde{P}_{k-1}^{n}\left(T_{k}\right)\right)+\epsilon\left(\beta \widetilde{E}_{k}^{n}\left(T_{k}\right)+\widetilde{P}_{k}^{n}\left(T_{k}\right)\right) \\
\tilde{\eta}_{k, k-1}^{n}=(1-\epsilon)\left(-\beta \widetilde{H}_{k-1}^{n}\left(T_{k}\right)-\widetilde{Q}_{k-1}^{n}\left(T_{k}\right)\right)+\epsilon\left(-\beta \widetilde{H}_{k}^{n}\left(T_{k}\right)-\widetilde{Q}_{k}^{n}\left(T_{k}\right)\right), \\
\tilde{\mu}_{k, k+1}^{0}=\mu_{k, k+1}^{0}-\left(\beta E\left(T_{k+1}\right)-P\left(T_{k+1}\right)\right) \\
\tilde{\eta}_{k, k+1}^{0}=\eta_{k, k+1}^{0}-\left(\beta H\left(T_{k+1}\right)-Q\left(T_{k+1}\right)\right) \\
\tilde{\mu}_{k, k-1}^{0}=\mu_{k, k-1}^{0}-\left(\beta E\left(T_{k}\right)+P\left(T_{k}\right)\right) \\
\tilde{\eta}_{k, k-1}^{0}=\eta_{k, k-1}^{0}+\left(\beta H\left(T_{k}\right)+Q\left(T_{k}\right)\right) .
\end{gathered}
$$


We shall prove the following convergence result:

Theorem 5.1. Let $\beta>0$. Then

(a) for any $\epsilon \in[0,1)$

$$
\begin{aligned}
\left.\nu \wedge \widetilde{P}_{k}^{n}\right|_{\Sigma_{k}} \rightarrow 0 \text { strongly in } \mathcal{L}_{\tau}^{2}\left(\Sigma_{k}\right), k=0, \ldots, K \\
\left(\widetilde{P}_{K}^{n}, \widetilde{Q}_{K}^{n}\right) \rightarrow 0 \text { in } C\left(I_{K} ; \mathcal{H}\right) \\
\left(\widetilde{E}_{0}^{n}, \widetilde{H}_{0}^{n}\right) \rightarrow 0 \text { in } C\left(I_{0} ; \mathcal{H}\right) \\
\left.\nu \wedge \widetilde{E}_{0}^{n}\right|_{\Sigma_{0}} \rightarrow 0 \text { strongly in } \mathcal{L}_{\tau}^{2}\left(\Sigma_{0}\right) .
\end{aligned}
$$

(b) For any $\epsilon \in(0,1)$ and for $k=0, \ldots, K$,

$$
\begin{gathered}
\left(\widetilde{E}_{k}^{n}, \widetilde{H}_{k}^{n}\right) \rightarrow 0 \text { and }\left(\widetilde{P}_{k}^{n}, \widetilde{Q}_{k}^{n}\right) \rightarrow 0 \text { in } C\left(I_{k} ; \mathcal{H}\right) \\
\left.\nu \wedge \widetilde{E}_{k}^{n}\right|_{\Sigma_{k}} \rightarrow 0 \text { strongly in } \mathcal{L}_{\tau}^{2}\left(\Sigma_{k}\right) .
\end{gathered}
$$

Remark 5.1. Part $(\mathrm{a})_{1,2}$ states that for any $\epsilon \in[0,1)$ the effective local optimal controls $\left\{\left.\nu \wedge P_{k}^{n}\right|_{\Sigma_{k}}\right\}_{k=0}^{K}$ converge strongly to the global optimal control $\left.\nu \wedge P\right|_{\Sigma}$, and the deviation $\left\|\left(E_{K}^{n}(T), H_{K}^{n}(T)\right)-\left(E_{T}, H_{T}\right)\right\|_{\mathcal{H}}$ of the local optimal trajectory $\left(E_{K}^{n}, H_{K}^{n}\right)$ at time $T$ from the target state $\left(E_{T}, H_{T}\right)$ converges to the deviation $\left\|(E(T), H(T))-\left(E_{T}, H_{T}\right)\right\|_{\mathcal{H}}$ of the global optimal trajectory at time $T$ from the target state. An a posteriori estimate of the error in the approximation is derived in the next section.

Remark 5.2. When $\epsilon=0$ it is possible to show that

$$
\begin{aligned}
& \left\{\begin{array}{l}
\left(\widetilde{E}_{k}^{n}, \widetilde{H}_{k}^{n}\right) \rightarrow 0 \text { weakly* in } L^{\infty}(0, T ; \mathcal{H}) \\
\left.\nu \wedge \widetilde{E}_{k}^{n}\right|_{\Sigma_{k}} \rightarrow 0 \text { weakly in } L_{\tau}^{2}\left(\Sigma_{k}\right),
\end{array}\right. \\
& \left(\widetilde{P}_{k}^{n}, \widetilde{Q}_{k}^{n}\right) \rightarrow 0 \text { weakly* in } L^{\infty}(0, T ; \mathcal{H}), k=0, \ldots, K-1
\end{aligned}
$$

provided the following backwards uniqueness property is valid: if $(E, H)$ satisfies the Maxwell system $(2.1)_{1,2}$ with $F=G=0$ and the boundary condition $(2.1)_{3}$ with $J=0$, and if $E(T)=H(T)=0$, then $E(0)=H(0)=0$. Whether or not this uniqueness property holds seems to be an open question.

Proof of Theorem 5.1. Although the technical details differ, the proof given below is structurally similar to proofs given in earlier works such as $[3-5]$, and in papers by the present authors (see, e.g. $[8,10]$ and references therein), all of which dealt with space domain decomposition of either direct or optimal control problems of one type or another. A key role in the convergence proofs in all of the papers is played by a fundamental recursion formula such as (5.22) below.

Set $\mathcal{X}=\mathcal{H}^{2 K}$ with the standard product norm. Let

$$
X=\left\{\left(\mu_{k, k+1}, \eta_{k, k+1}\right)_{k=0}^{K-1},\left(\mu_{k, k-1}, \eta_{k, k-1}\right)_{k=1}^{K}\right\} \in \mathcal{X}
$$

and let $\left(E_{k}, H_{k}\right),\left(P_{k}, Q_{k}\right)$ be the solution of $(4.8-4.10,4.6)$ with the superscripts $n+1$ and $n$ removed and with zero data

$$
F_{k}=G_{k}=0, \quad \phi=\psi=E_{T}=H_{T}=0 .
$$

Define the linear mapping $\mathcal{T}: \mathcal{X} \mapsto \mathcal{X}$ by

$$
\begin{aligned}
& \mathcal{T} X=\left\{\left(\beta E_{k+1}\left(T_{k+1}\right)-P_{k+1}\left(T_{k+1}\right), \beta H_{k+1}\left(T_{k+1}\right)-\right.\right. Q k+1 \\
&\left.\left(T_{k+1}\right)\right)_{k=0}^{K-1} \\
&\left.\left(\beta E_{k-1}\left(T_{k}\right)+P_{k-1}\left(T_{k}\right),-\beta H_{k-1}\left(T_{k}\right)-Q_{k-1}\left(T_{k}\right)\right)_{k=1}^{K}\right\} .
\end{aligned}
$$


Note that $X$ is a fixed point of $\mathcal{T}$ if and only if the transmission conditions (4.4) are satisfied, that is, if and only if $\left\{\left(E_{k}, H_{k}\right),\left(P_{k}, Q_{k}\right)\right\}_{k=0}^{K}$ is the solution of the global optimality system with vanishing data (5.9). Since, in this case, the optimal control is obviously $J_{\mathrm{opt}}=0$, it follows that the only fixed point of $\mathcal{T}$ is $X=0$.

The significance of the map $\mathcal{T}$ is that, if we set

$$
X^{n}=\left\{\left(\tilde{\mu}_{k, k+1}^{n-1}, \tilde{\eta}_{k, k+1}^{n-1}\right)_{k=0}^{K-1},\left(\tilde{\mu}_{k, k-1}^{n-1}, \tilde{\eta}_{k, k-1}^{n-1}\right)_{k=1}^{K}\right\}
$$

and let $\left\{\left(\widetilde{E}_{k}^{n}, \widetilde{H}_{k}^{n}\right),\left(\widetilde{P}_{k}^{n}, \widetilde{Q}_{k}^{n}\right)\right\}_{k=0}^{K}$ be the solution of (5.2-5.5) with $n$ replaced by $n-1$, then

$$
\begin{aligned}
& X^{n}=\left\{\left(\beta \widetilde{E}_{k}^{n}\left(T_{k+1}\right)-\widetilde{P}_{k}^{n}\left(T_{k+1}\right), \beta \widetilde{H}_{k}^{n}\left(T_{k+1}\right)-\widetilde{Q}_{k}^{n}\left(T_{k+1}\right)\right)_{k=0}^{K-1},\left(\beta \widetilde{E}_{k}^{n}\left(T_{k}\right)+\widetilde{P}_{k}^{n}\left(T_{k}\right),-\beta \widetilde{H}_{k}^{n}\left(T_{k}\right)-\widetilde{Q}_{k}^{n}\left(T_{k}\right)\right)_{k=1}^{K}\right\} \\
& \mathcal{T} X^{n}=\left\{\left(\beta \widetilde{E}_{k+1}^{n}\left(T_{k+1}\right)-\widetilde{P}_{k+1}^{n}\left(T_{k+1}\right), \beta \widetilde{H}_{k+1}^{n}\left(T_{k+1}\right)-\widetilde{Q}_{k+1}^{n}\left(T_{k+1}\right)\right)_{k=0}^{K-1},\right. \\
& \left.\left(\beta \widetilde{E}_{k-1}^{n}\left(T_{k}\right)+\widetilde{P}_{k-1}^{n}\left(T_{k}\right),-\beta \widetilde{H}_{k-1}^{n}\left(T_{k}\right)-\widetilde{Q}_{k-1}^{n}\left(T_{k}\right)\right)_{k=1}^{K}\right\}
\end{aligned}
$$

and the relaxed iteration step $(5.5,5.6)$ may be expressed as the relaxed fixed point iteration

$$
X^{n+1}=(1-\epsilon) \mathcal{T} X^{n}+\epsilon X^{n}:=\mathcal{T}_{\epsilon} X^{n} .
$$

The following result shows that $\mathcal{T}$ is nonexpansive.

Lemma 5.1. For any $X \in \mathcal{X}$,

$$
\|\mathcal{T} X\|_{\mathcal{X}}^{2}=\|X\|_{\mathcal{X}}^{2}-2 \mathcal{F}_{\beta}
$$

where

$$
\mathcal{F}_{\beta}=2 \beta \sum_{k=0}^{K} \int_{\Sigma_{k}}\left|\nu \wedge P_{k}\right|^{2} \mathrm{~d} \Sigma+2 \beta z\left\|\left(E_{K}\left(T_{K+1}\right), H_{K}\left(T_{K+1}\right)\right)\right\|_{\mathcal{H}}^{2} .
$$

Proof. We have

$$
\begin{aligned}
\|X\|_{\mathcal{X}}^{2}= & \sum_{k=0}^{K-1}\left\|\left(\beta E_{k}\left(T_{k+1}\right)-P_{k}\left(T_{k+1}\right), \beta H_{k}\left(T_{k+1}\right)-Q_{k}\left(T_{k+1}\right)\right)\right\|_{\mathcal{H}}^{2} \\
& +\sum_{k=1}^{K}\left\|\left(\beta E_{k}\left(T_{k}\right)+P_{k}\left(T_{k}\right),-\beta H_{k}\left(T_{k}\right)-Q_{k}\left(T_{k}\right)\right)\right\|_{\mathcal{H}}^{2} .
\end{aligned}
$$

Let us define

$$
\mathcal{E}_{k, \beta}(t)=\beta^{2}\left(\left\|E_{k}(t)\right\|_{\varepsilon}^{2}+\left\|H_{k}(t)\right\|_{\mu}^{2}\right)+\left\|P_{k}(t)\right\|_{\varepsilon}^{2}+\left\|Q_{k}(t)\right\|_{\mu}^{2} .
$$

One finds after a little calculation that (5.14) may be written

$$
\begin{aligned}
\|X\|_{\mathcal{X}}^{2}= & \sum_{k=1}^{K-1}\left[\mathcal{E}_{k, \beta}\left(T_{k+1}\right)+\mathcal{E}_{k, \beta}\left(T_{k}\right)\right]+\mathcal{E}_{0, \beta}\left(T_{1}\right)+\mathcal{E}_{K, \beta}\left(T_{K}\right) \\
& -2 \beta \sum_{k=0}^{K-1} \operatorname{Re}\left[\left\langle E_{k}(t), P_{k}(t)\right\rangle_{\varepsilon}+\left\langle H_{k}(t), Q_{k}(t)\right\rangle_{\mu}\right]_{t=T_{k}}^{T_{k+1}} \\
& +2 \beta \operatorname{Re}\left[\left\langle E_{K}\left(T_{K}\right), P_{K}\left(T_{K}\right)\right\rangle_{\varepsilon}+\left\langle H_{K}\left(T_{K}\right), Q_{K}\left(T_{K}\right)\right\rangle_{\mu}\right] .
\end{aligned}
$$


Form

$$
\begin{aligned}
0 & =\int_{T_{k}}^{T_{k+1}}\left[\left\langle\varepsilon E_{k}^{\prime}-\operatorname{rot} H_{k}+\sigma E_{k}, P_{k}\right\rangle+\left\langle\mu H_{k}^{\prime}+\operatorname{rot} E_{k}, Q_{k}\right\rangle\right] \mathrm{d} t \\
& =\left.\left\langle\left(E_{k}(t), H_{k}(t)\right),\left(P_{k}(t), Q_{k}(t)\right)\right\rangle_{\mathcal{H}}\right|_{t=T_{k}} ^{T_{k+1}}+\int_{\Sigma_{k}}\left|\nu \wedge P_{k}\right|^{2} \mathrm{~d} \Sigma,
\end{aligned}
$$

where we have used (3.3). In particular we have

$$
\begin{aligned}
\left\langle\left(E_{K}\left(T_{K}\right), H_{K}\left(T_{K}\right)\right),\left(P_{K}\right.\right. & \left.\left.\left(T_{K}\right), Q_{K}\left(T_{K}\right)\right)\right\rangle_{\mathcal{H}} \\
& =\left\langle\left(E_{K}\left(T_{K+1}\right), H_{K}\left(T_{K+1}\right)\right),\left(P_{K}\left(T_{K+1}\right), Q_{K}\left(T_{K+1}\right)\right)\right\rangle_{\mathcal{H}}+\int_{\Sigma_{K}}\left|\nu \wedge P_{K}\right|^{2} \mathrm{~d} \Sigma \\
& =z\left\|\left(E_{K}\left(T_{K+1}\right), H_{K}\left(T_{K+1}\right)\right)\right\|_{\mathcal{H}}^{2}+\int_{\Sigma_{K}}\left|\nu \wedge P_{K}\right|^{2} \mathrm{~d} \Sigma .
\end{aligned}
$$

It follows from $(5.15-5.17)$ that

$$
\|X\|_{\mathcal{X}}^{2}=\mathcal{E}_{\beta}+\mathcal{F}_{\beta}
$$

where

$$
\mathcal{E}_{\beta}=\sum_{k=1}^{K-1}\left[\mathcal{E}_{k, \beta}\left(T_{k+1}\right)+\mathcal{E}_{k, \beta}\left(T_{k}\right)\right]+\mathcal{E}_{0, \beta}\left(T_{1}\right)+\mathcal{E}_{K, \beta}\left(T_{K}\right) .
$$

A similar calculation yields

$$
\begin{aligned}
\|\mathcal{T} X\|_{\mathcal{X}}^{2}= & \sum_{k=0}^{K-1}\left\|\left(\beta E_{k+1}\left(T_{k+1}\right)-P_{k+1}\left(T_{k+1}\right), \beta H_{k+1}\left(T_{k+1}\right)-Q_{k+1}\left(T_{k+1}\right)\right)\right\|_{\mathcal{H}}^{2} \\
& +\sum_{k=1}^{K}\left\|\left(\beta E_{k-1}\left(T_{k}\right)+P_{k-1}\left(T_{k}\right),-\beta H_{k-1}\left(T_{k}\right)-Q_{k-1}\left(T_{k}\right)\right)\right\|_{\mathcal{H}}^{2} \\
= & \mathcal{E}_{\beta}+2 \beta \sum_{k=0}^{K-1} \operatorname{Re}\left[\left\langle E_{k}(t), P_{k}(t)\right\rangle_{\varepsilon}+\left\langle H_{k}(t), Q_{k}(t)\right\rangle_{\mu}\right]_{t=T_{k}}^{T_{k+1}} \\
& -2 \beta \operatorname{Re}\left[\left\langle E_{K}\left(T_{K}\right), P_{K}\left(T_{K}\right)\right\rangle_{\varepsilon}+\left\langle H_{K}\left(T_{K}\right), Q_{K}\left(T_{K}\right)\right\rangle_{\mu}\right] \\
= & \mathcal{E}_{\beta}-\mathcal{F}_{\beta} .
\end{aligned}
$$

Thus (5.12) follows from (5.18) and (5.20).

To continue with the proof of Theorem 5.1, we set

$$
\begin{aligned}
\mathcal{E}_{k, \beta}^{n}(t) & =\beta^{2}\left(\left\|\widetilde{E}_{k}^{n}(t)\right\|_{\varepsilon}^{2}+\left\|\widetilde{H}_{k}^{n}(t)\right\|_{\mu}^{2}\right)+\left\|\widetilde{P}_{k}^{n}(t)\right\|_{\varepsilon}^{2}+\left\|\widetilde{Q}_{k}^{n}(t)\right\|_{\mu}^{2} \\
\mathcal{E}_{\beta}^{n} & =\sum_{k=1}^{K-1}\left[\mathcal{E}_{k, \beta}^{n}\left(T_{k+1}\right)+\mathcal{E}_{k, \beta}^{n}\left(T_{k}\right)\right]+\mathcal{E}_{0, \beta}^{n}\left(T_{1}\right)+\mathcal{E}_{K, \beta}^{n}\left(T_{K}\right) \\
& =\sum_{k=0}^{K-1}\left[\mathcal{E}_{k, \beta}^{n}\left(T_{k+1}\right)+\mathcal{E}_{k+1, \beta}^{n}\left(T_{k+1}\right)\right] \\
\mathcal{F}_{\beta}^{n} & =2 \beta \sum_{k=0}^{K} \int_{\Sigma_{k}}\left|\nu \wedge \widetilde{P}_{k}^{n}\right|^{2} \mathrm{~d} \Sigma+2 \beta z\left\|\left(\widetilde{E}_{K}^{n}\left(T_{K+1}\right), \widetilde{H}_{K}^{n}\left(T_{K+1}\right)\right)\right\|_{\mathcal{H}}^{2} .
\end{aligned}
$$


From (5.18) and (5.20) we then have

$$
\left\|X^{n}\right\|_{\mathcal{X}}^{2}=\mathcal{E}_{\beta}^{n}+\mathcal{F}_{\beta}^{n}, \quad\left\|\mathcal{T} X^{n}\right\|_{\mathcal{X}}^{2}=\mathcal{E}_{\beta}^{n}-\mathcal{F}_{\beta}^{n} .
$$

Lemma 5.2. For any $\epsilon \in[0,1)$ we have

$$
\mathcal{E}_{\beta}^{n+1}+\mathcal{F}_{\beta}^{n+1} \leq \mathcal{E}_{\beta}^{n}-(1-2 \epsilon) \mathcal{F}_{\beta}^{n} .
$$

Proof. As the relaxed iteration step $(5.5,5.6)$ may be expressed as the relaxed fixed point iteration $(5.11)$, from Lemma 5.1 and (5.21) we have

$$
\begin{aligned}
\left\|X^{n+1}\right\|_{\mathcal{X}}^{2} & =\mathcal{E}_{\beta}^{n+1}+\mathcal{F}_{\beta}^{n+1} \\
& =\left((1-\epsilon)^{2}+\epsilon^{2}\right)\left\|X^{n}\right\|_{\mathcal{X}}^{2}-2(1-\epsilon)^{2} \mathcal{F}_{\beta}^{n}+2 \epsilon(1-\epsilon) \operatorname{Re}\left(\mathcal{T} X^{n}, X^{n}\right)_{\mathcal{X}} \\
& =\left((1-\epsilon)^{2}+\epsilon^{2}\right) \mathcal{E}_{\beta}^{n}-(1-2 \epsilon) \mathcal{F}_{\beta}^{n}+2 \epsilon(1-\epsilon) \operatorname{Re}\left(\mathcal{T} X^{n}, X^{n}\right)_{\mathcal{X}} .
\end{aligned}
$$

From (5.21) and the Cauchy-Schwarz inequality we have

$$
\left|\left(\mathcal{T} X^{n}, X^{n}\right)_{\mathcal{X}}\right| \leq \sqrt{\left(\mathcal{E}_{\beta}^{n}\right)^{2}-\left(\mathcal{F}_{\beta}^{n}\right)^{2}} \leq \mathcal{E}_{\beta}^{n} .
$$

Lemma 5.2 follows immediately from (5.23) and (5.24).

By iterating (5.22) down to $n=1$ we obtain

$$
\mathcal{E}_{\beta}^{n+1} \leq \mathcal{E}_{\beta}^{1}-\sum_{p=1}^{n+1} c_{p}(\epsilon) \mathcal{F}_{\beta}^{p},
$$

where

$$
c_{p}(\epsilon)=2(1-\epsilon), p=2, \ldots, n ; \quad c_{1}(\epsilon)=1-2 \epsilon, c_{n+1}(\epsilon)=1 .
$$

The crucial inequality (5.25) implies, in particular, that $\mathcal{F}_{\beta}^{n} \rightarrow 0$ as $n \rightarrow \infty$, hence

$$
\begin{gathered}
\left.\nu \wedge \widetilde{P}_{k}^{n}\right|_{\Sigma_{k}} \rightarrow 0 \text { strongly in } \mathcal{L}_{\tau}^{2}\left(\Sigma_{k}\right), k=0, \ldots, K \\
\left(\widetilde{E}_{K}^{n}\left(T_{K+1}\right), \widetilde{H}_{K}^{n}\left(T_{K+1}\right)\right) \rightarrow 0 \text { strongly in } \mathcal{H} .
\end{gathered}
$$

From (5.27) and (5.4) 2 we then obtain

$$
\left(\widetilde{P}_{K}^{n}\left(T_{K+1}\right), \widetilde{Q}_{K}^{n}\left(T_{K+1}\right)\right) \rightarrow 0 \text { strongly in } \mathcal{H} .
$$

Since, for the solution of (2.1) (with $F=G=0)$ the mapping taking $((\phi, \psi), J)$ to $\left((E, H),\left.\nu \wedge E\right|_{\Sigma}\right)$ is continuous from $\mathcal{H} \times \mathcal{L}_{\tau}^{2}(\Sigma)$ into $L^{\infty}(0, T ; \mathcal{H}) \times \mathcal{L}_{\tau}^{2}(\Sigma)$, it follows from $(5.26)$ and $\widetilde{E}_{0}^{n}(0)=\widetilde{H}_{0}^{n}(0)=0$, that

$$
\begin{aligned}
& \left(\widetilde{E}_{0}^{n}, \widetilde{H}_{0}^{n}\right) \rightarrow 0 \text { strongly in } C\left(I_{0} ; \mathcal{H}\right) \\
& \left.\nu \wedge \widetilde{E}_{0}^{n}\right|_{\Sigma_{0}} \rightarrow 0 \text { strongly in } \mathcal{L}_{\tau}^{2}\left(\Sigma_{0}\right) .
\end{aligned}
$$

Similarly, for the solution of (2.5) the mapping from the final data $(P(T), Q(T))$ to $\left((P, Q),\left.\nu \wedge P\right|_{\Sigma}\right)$ is continuous $\mathcal{H} \mapsto L^{\infty}(0, T ; \mathcal{H}) \times \mathcal{L}_{\tau}^{2}(\Sigma)$ so, in particular, we obtain from $(5.28)$

$$
\left(\widetilde{P}_{K}^{n}, \widetilde{Q}_{K}^{n}\right) \rightarrow 0 \text { strongly in } C\left(I_{K} ; \mathcal{H}\right) .
$$

This proves part (a) of Theorem 5.1. 
To prove part (b) of Theorem 5.1 we invoke the following result:

Proposition 5.1. (Schaefer [17]) Let $\mathcal{C}$ be a closed convex set in a uniformly convex Banach space $\mathcal{X}$ having a weakly continuous duality mapping, and let $\mathcal{T}: \mathcal{C} \mapsto \mathcal{C}$ be a nonexpansive mapping with at least one fixed point. Set $\mathcal{T}_{\epsilon}=\epsilon I+(1-\epsilon) \mathcal{T}$. Then for any $\epsilon \in(0,1)$ the mapping $\mathcal{T}_{\epsilon}$ is asymptotically regular, that is to say,

$$
\left\|\mathcal{T}_{\epsilon}^{n+1} X-\mathcal{T}_{\epsilon}^{n} X\right\|_{\mathcal{X}} \rightarrow 0, \quad \forall X \in \mathcal{C} .
$$

It follows from Proposition 5.1 that $\left\|X^{n+1}-X^{n}\right\|_{\mathcal{X}} \rightarrow 0$ or, equivalently,

$$
\left\|\mathcal{T} X^{n}-X^{n}\right\|_{\mathcal{X}} \rightarrow 0
$$

since $X^{n+1}-X^{n}=(1-\epsilon)\left(\mathcal{T} X^{n}-X^{n}\right)$. We have

$$
\begin{gathered}
\mathcal{T} X^{n}-X^{n}=\left\{\left(\beta\left(\widetilde{E}_{k+1}^{n}\left(T_{k+1}\right)-\widetilde{E}_{k}^{n}\left(T_{k+1}\right)\right)-\left(\widetilde{P}_{k+1}^{n}\left(T_{k+1}\right)-\widetilde{P}_{k}^{n}\left(T_{k+1}\right)\right),\right.\right. \\
\left.\beta\left(\widetilde{H}_{k+1}^{n}\left(T_{k+1}\right)-\widetilde{H}_{k}^{n}\left(T_{k+1}\right)\right)-\left(\widetilde{Q}_{k+1}^{n}\left(T_{k+1}\right)-\widetilde{Q}_{k}^{n}\left(T_{k+1}\right)\right)\right)_{k=0}^{K-1}, \\
\left(\beta\left(\widetilde{E}_{k-1}^{n}\left(T_{k}\right)-\widetilde{E}_{k}^{n}\left(T_{k}\right)\right)+\left(\widetilde{P}_{k-1}^{n}\left(T_{k}\right)-\widetilde{P}_{k}^{n}\left(T_{k}\right)\right),\right. \\
\left.\left.-\beta\left(\widetilde{H}_{k-1}^{n}\left(T_{k}\right)-\widetilde{H}_{k}^{n}\left(T_{k}\right)\right)-\left(\widetilde{Q}_{k-1}^{n}\left(T_{k}\right)-\widetilde{Q}_{k}^{n}\left(T_{k}\right)\right)\right)_{k=1}^{K}\right\} .
\end{gathered}
$$

A straightforward calculation shows that

$$
\begin{aligned}
\left\|\mathcal{T} X^{n}-X^{n}\right\|_{\mathcal{X}}^{2}= & 2 \sum_{k=0}^{K-1}\left\{\beta^{2}\left\|\left(\widetilde{E}_{k+1}^{n}\left(T_{k+1}\right)-\widetilde{E}_{k}^{n}\left(T_{k+1}\right), \widetilde{H}_{k+1}^{n}\left(T_{k+1}\right)-\widetilde{H}_{k}^{n}\left(T_{k+1}\right)\right)\right\|_{\mathcal{H}}^{2}\right. \\
& \left.+\left\|\left(\widetilde{P}_{k+1}^{n}\left(T_{k+1}\right)-\widetilde{P}_{k}^{n}\left(T_{k+1}\right), \widetilde{Q}_{k+1}^{n}\left(T_{k+1}\right)-\widetilde{Q}_{k}^{n}\left(T_{k+1}\right)\right)\right\|_{\mathcal{H}}^{2}\right\}
\end{aligned}
$$

Therefore, for $k=0, \ldots, K-1$,

$$
\begin{aligned}
& \left\|\left(\widetilde{E}_{k+1}^{n}\left(T_{k+1}\right)-\widetilde{E}_{k}^{n}\left(T_{k+1}\right), \widetilde{H}_{k+1}^{n}\left(T_{k+1}\right)-\widetilde{H}_{k}^{n}\left(T_{k+1}\right)\right)\right\|_{\mathcal{H}} \rightarrow 0 \\
& \left\|\left(\widetilde{P}_{k+1}^{n}\left(T_{k+1}\right)-\widetilde{P}_{k}^{n}\left(T_{k+1}\right), \widetilde{Q}_{k+1}^{n}\left(T_{k+1}\right)-\widetilde{Q}_{k}^{n}\left(T_{k+1}\right)\right)\right\|_{\mathcal{H}} \rightarrow 0 .
\end{aligned}
$$

By what has already been proved we have

$$
\left(\widetilde{P}_{K}^{n}, \widetilde{Q}_{K}^{n}\right) \rightarrow 0 \text { in } C\left(I_{K} ; \mathcal{H}\right)
$$

so that, in particular,

$$
\left\|\left(\widetilde{P}_{K}^{n}\left(T_{K}\right), \widetilde{Q}_{K}^{n}\left(T_{K}\right)\right)\right\|_{\mathcal{H}} \rightarrow 0 .
$$

It then follows from (5.35) with $k=K-1$ that

$$
\left\|\left(\widetilde{P}_{K-1}^{n}\left(T_{K}\right), \widetilde{Q}_{K-1}^{n}\left(T_{K}\right)\right)\right\|_{\mathcal{H}} \rightarrow 0,
$$

from which follows that

$$
\left(\widetilde{P}_{K-1}^{n}, \widetilde{Q}_{K-1}^{n}\right) \rightarrow 0 \text { in } C\left(I_{K-1} ; \mathcal{H}\right)
$$

and, in particular, that

$$
\left\|\left(\widetilde{P}_{K-1}^{n}\left(T_{K-1}\right), \widetilde{Q}_{K-1}^{n}\left(T_{K-1}\right)\right)\right\|_{\mathcal{H}} \rightarrow 0 .
$$


We may now repeat the argument to conclude that

$$
\left(\widetilde{P}_{k}^{n}, \widetilde{Q}_{k}^{n}\right) \rightarrow 0 \text { in } C\left(I_{k} ; \mathcal{H}\right), k=K, K-1, \ldots, 0 .
$$

In a similar way, since we have already proved that

$$
\left(\widetilde{E}_{0}^{n}, \widetilde{H}_{0}^{n}\right) \rightarrow 0 \text { in } C\left(I_{0} ; \mathcal{H}\right)
$$

we have

$$
\left\|\left(\widetilde{E}_{0}^{n}\left(T_{1}\right), \widetilde{H}_{0}^{n}\left(T_{1}\right)\right)\right\|_{\mathcal{H}} \rightarrow 0 .
$$

Use of (5.34) with $k=0$ gives

$$
\left\|\left(\widetilde{E}_{1}^{n}\left(T_{1}\right), \widetilde{H}_{1}^{n}\left(T_{1}\right)\right)\right\|_{\mathcal{H}} \rightarrow 0,
$$

hence

$$
\begin{gathered}
\left(\widetilde{E}_{1}^{n}, \widetilde{H}_{1}^{n}\right) \rightarrow 0 \text { in } C\left(I_{1} ; \mathcal{H}\right) \\
\left.\nu \wedge \widetilde{E}_{1}^{n}\right|_{\Sigma_{1}} \rightarrow 0 \text { strongly in } \mathcal{L}_{\tau}^{2}\left(\Sigma_{1}\right),
\end{gathered}
$$

and so forth. This completes the proof of Theorem 5.1.

\section{A POSTERIORI ESTIMATES}

The purpose of this section is to derive a posteriori estimates of the difference between the solutions of the local optimality systems and the solution of the global optimality system in terms of the mismatch of the $n$-th iterates, or of successive iterates, across the interfaces $t=T_{k}, k=1, \ldots, K$.

We introduce the error at the $n$-th iteration, $\mathrm{e}^{n}$, by

$$
\begin{aligned}
\mathrm{e}^{n}:= & \max _{0 \leq k \leq K}\left\|\left(\widetilde{E}_{k}^{n}, \widetilde{H}_{k}^{n}\right)\right\|_{L^{\infty}\left(I_{k} ; \mathcal{H}\right)}^{2}+\max _{0 \leq k \leq K}\left\|\left(\widetilde{P}_{k}^{n}, \widetilde{Q}_{k}^{n}\right)\right\|_{L^{\infty}\left(I_{k} ; \mathcal{H}\right)}^{2} \\
& +\sum_{k=0}^{K} \int_{\Sigma_{k}}\left\{\alpha\left(\left|\nu \wedge \widetilde{E}_{k}^{n}\right|^{2}+\left|\nu \wedge \widetilde{P}_{k}^{n}\right|^{2}\right)+\frac{1}{\alpha}\left(\left|\widetilde{H}_{k \tau}^{n}\right|^{2}+\left|\widetilde{Q}_{k \tau}^{n}\right|^{2}\right)\right\} \mathrm{d} \Sigma
\end{aligned}
$$

and further define

$$
\begin{gathered}
\mathrm{e}_{k}^{n}(t)=\left\|\left(\widetilde{E}_{k}^{n}(t), \widetilde{H}_{k}^{n}(t)\right)\right\|_{\mathcal{H}}^{2}+\int_{T_{k}}^{t} \int_{\Gamma}\left(\alpha\left|\nu \wedge \widetilde{E}_{k}^{n}\right|^{2}+\frac{1}{\alpha}\left|\widetilde{H}_{k \tau}^{n}\right|^{2}\right) \mathrm{d} \Gamma \mathrm{d} t \\
+\left\|\left(\widetilde{P}_{k}^{n}(t), \widetilde{Q}_{k}^{n}(t)\right)\right\|_{\mathcal{H}}^{2}+\int_{t}^{T_{k+1}} \int_{\Gamma}\left(\alpha\left|\nu \wedge \widetilde{P}_{k}^{n}\right|^{2}+\frac{1}{\alpha}\left|\widetilde{Q}_{k \tau}^{n}\right|^{2}\right) \mathrm{d} \Gamma \mathrm{d} t \\
\mathrm{e}^{n, n+1}=\max _{0 \leq k \leq K}\left\|\mathrm{e}_{k}^{n}+\mathrm{e}_{k}^{n+1}\right\|_{L^{\infty}(0, T)},
\end{gathered}
$$

which is a measure of the cumulative errors at the $n$-th and $(n+1)$-st iterations combined. In what follows we shall obtain a posteriori estimates of $\mathrm{e}^{n}$ and $\mathrm{e}^{n, n+1}$ in terms of the quantities

$$
\begin{aligned}
\mathcal{E}_{k, k+1}^{n}\left(T_{k+1}\right)= & \left\|\left(E_{k}^{n}\left(T_{k+1}\right)-E_{k+1}^{n}\left(T_{k+1}\right), H_{k}^{n}\left(T_{k+1}\right)-H_{k+1}^{n}\left(T_{k+1}\right)\right)\right\|_{\mathcal{H}}^{2} \\
& +\left\|\left(P_{k}^{n}\left(T_{k+1}\right)-P_{k+1}^{n}\left(T_{k+1}\right), Q_{k}^{n}\left(T_{k+1}\right)-Q_{k+1}^{n}\left(T_{k+1}\right)\right)\right\|_{\mathcal{H}}^{2}
\end{aligned}
$$


and

$$
\begin{aligned}
\mathcal{E}_{k, k+1}^{n, n+1}\left(T_{k+1}\right)= & \left.\| E_{k}^{n}\left(T_{k+1}\right)-E_{k+1}^{n+1}\left(T_{k+1}\right), H_{k}^{n}\left(T_{k+1}\right)-H_{k+1}^{n+1}\left(T_{k+1}\right)\right) \|_{\mathcal{H}}^{2} \\
& +\left\|\left(P_{k}^{n}\left(T_{k+1}\right)-P_{k+1}^{n+1}\left(T_{k+1}\right), Q_{k}^{n}\left(T_{k+1}\right)-Q_{k+1}^{n+1}\left(T_{k+1}\right)\right)\right\|_{\mathcal{H}}^{2},
\end{aligned}
$$

which are, respectively, measures of the mismatch of the $n$-th iterates, and of successive iterates, across the break point $t=T_{k+1}$. The main results of this section are the following error estimates.

Theorem 6.1. Let $\epsilon \in[0,1)$. Then

$$
\mathrm{e}^{n} \leq C \sqrt{\mathcal{E}_{\beta}^{n}+\mathcal{F}_{\beta}^{n}}\left\{\sum_{k=0}^{K-1} \mathcal{E}_{k, k+1}^{n}\left(T_{k+1}\right)\right\}^{1 / 2}
$$

where

$$
C=\sqrt{2} \max (1,1 / \beta)\left(2+\max \left(\left\|\alpha^{-1}\right\|_{L^{\infty}(\Gamma)}, \kappa\right)\right) .
$$

Theorem 6.2. Let $\epsilon \in[0,1 / 2)$. Then

$$
\mathrm{e}^{n, n+1} \leq C_{\epsilon} \sqrt{\mathcal{E}_{\beta}^{n+1}+\mathcal{E}_{\beta}^{n}}\left\{\sum_{k=0}^{K-1}\left(\mathcal{E}_{k, k+1}^{n, n+1}\left(T_{k+1}\right)+\mathcal{E}_{k+1, k}^{n, n+1}\left(T_{k+1}\right)\right)\right\}^{1 / 2}
$$

where

$$
C_{\epsilon}=\sqrt{2} \max (1,1 / \beta)\left(2+(1-2 \epsilon)^{-1} \max \left(\left\|\alpha^{-1}\right\|_{L^{\infty}(\Gamma)}, \kappa\right)\right) .
$$

It shall be shown below that $\mathcal{E}_{\beta}^{n}+\mathcal{F}_{\beta}^{n} \leq \tilde{C}$ with a constant $\tilde{C}$ that is explicitly computable in terms of $\beta, K$ and the input data to the global and local optimal control problems. We therefore have the following corollaries:

Corollary 6.1. Let $\epsilon \in[0,1)$. There is an explicitly computable constant $C$, depending only on $\beta, K$ and the input data to the global and local optimal control problems, such that

$$
\mathrm{e}^{n} \leq C\left\{\sum_{k=0}^{K-1} \mathcal{E}_{k, k+1}^{n}\left(T_{k+1}\right)\right\}^{1 / 2}
$$

Corollary 6.2. Let $\epsilon \in[0,1 / 2)$. There is an explicitly computable constant $C_{\epsilon}$, depending only on $\epsilon, \beta, K$ and the input data to the global and local optimal control problems, such that

$$
\mathrm{e}^{n, n+1} \leq C_{\epsilon}\left\{\sum_{k=0}^{K-1}\left(\mathcal{E}_{k, k+1}^{n, n+1}\left(T_{k+1}\right)+\mathcal{E}_{k+1, k}^{n, n+1}\left(T_{k+1}\right)\right)\right\}^{1 / 2} .
$$

Remark 6.1. The constant $C$, which is independent of $\epsilon$, and $C_{\epsilon}$ will be calculated in the proofs of Corollaries 6.1 and 6.2. The estimate (6.4) says that if the $n$-th iterates are nearly equal across the interfaces $t=T_{k+1}, k=0, \ldots, K-1$, then the error at the $n$ iteration is nearly zero. It thus provides a stopping criterion for the algorithm in terms of the mismatch of the $n$ iterates at the break points. Similarly, equation (6.5) provides a stopping criterion in terms of the differences of successive iterates at the break points.

Remark 6.2. The above error estimates are further important for future work on adaptive finite element discretizations. The errors bewteen the global solution and the local solutions to the discretized subsystems 
will be given in terms of the residuals with respect to solving the system equations at a given iteration and the estimates above. It will be possible to decide, based on a posteriori information, which of the errors is dominant, and whether a refinement of the discretization in one of the subsystems will be necessary. This will also be true for similar estimates on the spatial domain decomposition. Thus, the ultimate goal will be to develop space-time adaptive schemes based on error estimates of the type given above.

Theorem 6.1 is a consequence of the following two lemmas:

Lemma 6.1. Let $\epsilon \in[0,1)$. Then

$$
\mathcal{F}_{\beta}^{n} \leq \sqrt{2} \max (1, \beta)\left(\mathcal{E}_{\beta}^{n}+\mathcal{F}_{\beta}^{n}\right)^{1 / 2}\left\{\sum_{k=0}^{K-1} \mathcal{E}_{k, k+1}^{n}\left(T_{k+1}\right)\right\}^{1 / 2} .
$$

Lemma 6.2. Let $\epsilon \in[0,1)$. Then

$$
\mathrm{e}^{n} \leq 2 \sqrt{2} \max (1,1 / \beta) \sqrt{\mathcal{E}_{\beta}^{n}}\left\{\sum_{k=0}^{K-1} \mathcal{E}_{k, k+1}^{n}\left(T_{k+1}\right)\right\}^{1 / 2}+\frac{1}{\beta} \mathcal{F}_{\beta}^{n} \max \left(\left\|\alpha^{-1}\right\|_{L^{\infty}(\Gamma)}, z\right) .
$$

Similarly, Theorem 6.2 follows immediately from the following two results:

Lemma 6.3. Let $\epsilon \in[0,1)$. Then

$$
\mathcal{F}_{\beta}^{n+1}+(1-2 \epsilon) \mathcal{F}_{\beta}^{n} \leq \sqrt{2} \max (1, \beta) \sqrt{\mathcal{E}_{\beta}^{n+1}+\mathcal{E}_{\beta}^{n}}\left\{\sum_{k=0}^{K-1}\left(\mathcal{E}_{k, k+1}^{n, n+1}\left(T_{k+1}\right)+\mathcal{E}_{k+1, k}^{n, n+1}\left(T_{k+1}\right)\right)\right\}^{1 / 2}
$$

Lemma 6.4. We have

$$
\begin{aligned}
\mathrm{e}^{n, n+1} \leq & \frac{1}{\beta} \max \left(\left\|\alpha^{-1}\right\|_{L^{\infty}(\Gamma)}, z\right)\left(\mathcal{F}_{\beta}^{n+1}+\mathcal{F}_{\beta}^{n}\right) \\
& +\sqrt{2} \max (1,1 / \beta) \sqrt{\mathcal{E}_{\beta}^{n+1}+\mathcal{E}_{\beta}^{n}}\left\{\sum_{k=0}^{K-1}\left(\mathcal{E}_{k, k+1}^{n, n+1}\left(T_{k+1}\right)+\mathcal{E}_{k+1, k}^{n, n+1}\left(T_{k+1}\right)\right)\right\}^{1 / 2} .
\end{aligned}
$$

Proof of Lemma 6.1. We start with the fundamental recursion (5.22), which we write as

$$
2(1-\epsilon) \mathcal{F}_{\beta}^{n} \leq\left(\mathcal{E}_{\beta}^{n}+\mathcal{F}_{\beta}^{n}\right)-\left(\mathcal{E}_{\beta}^{n+1}+\mathcal{F}_{\beta}^{n+1}\right) .
$$

From (5.21) the right side of (6.10) may be written

$$
\begin{aligned}
\left\|X^{n}\right\|_{\mathcal{X}}^{2}-\left\|X^{n+1}\right\|_{\mathcal{X}}^{2} & \leq\left\|X^{n}-X^{n+1}\right\|_{\mathcal{X}}\left(\left\|X^{n}\right\|_{\mathcal{X}}+\left\|X^{n+1}\right\|_{\mathcal{X}}\right) \\
& \leq 2(1-\epsilon)\left\|X^{n}-\mathcal{T} X^{n}\right\|_{\mathcal{X}}\left\|^{n}\right\|_{\mathcal{X}}
\end{aligned}
$$

since $\left\{\left\|X^{n}\right\|_{\mathcal{X}}\right\}$ is a nonincreasing sequence. Since

$$
\widetilde{E}_{k+1}^{n}\left(T_{k+1}\right)-\widetilde{E}_{k}^{n}\left(T_{k+1}\right)=E_{k+1}^{n}\left(T_{k+1}\right)-E_{k}^{n}\left(T_{k+1}\right)
$$


and, similarly, for the other components, it follows from $(6.10,6.11)$ and $(5.33)$ that

$$
\mathcal{F}_{\beta}^{n} \leq \sqrt{2}\left\|X^{n}\right\|_{\mathcal{X}} \max (1, \beta)\left\{\sum_{k=0}^{K-1} \mathcal{E}_{k, k+1}^{n}\left(T_{k+1}\right)\right\}^{1 / 2}
$$

which is the conclusion of Lemma 6.1.

Proof of Lemma 6.2. The starting point is the following identity for the solution of (2.1) with $F=G=0$, which may be proved by standard energy methods (see [11]):

$$
\begin{aligned}
\|(E(t), H(t))\|_{\mathcal{H}}^{2} & +2 \int_{0}^{t} \int_{\Omega} \sigma E \cdot E \mathrm{~d} x \mathrm{~d} t+\int_{0}^{t} \int_{\Gamma}\left(\alpha|\nu \wedge E|^{2}+\frac{1}{\alpha}\left|H_{\tau}\right|^{2}\right) \mathrm{d} \Gamma \mathrm{d} t=\|(\phi, \psi)\|_{\mathcal{H}}^{2} \\
& +\int_{0}^{t} \int_{\Gamma} \frac{1}{\alpha}|J|^{2} \mathrm{~d} \Gamma \mathrm{d} t .
\end{aligned}
$$

This identity is valid for $0 \leq t \leq T,(\phi, \psi) \in \mathcal{H}$ and $J \in \mathcal{L}_{\tau}^{2}(\Sigma)$. We apply (6.12) to the solution $\left(\widetilde{E}_{k}^{n}, \widetilde{H}_{k}^{n}\right)$ on the interval $I_{k}$ to obtain

$$
\begin{aligned}
\left\|\left(\widetilde{E}_{k}^{n}(t), \widetilde{H}_{k}^{n}(t)\right)\right\|_{\mathcal{H}}^{2} & +\int_{T_{k}}^{t} \int_{\Gamma}\left(\alpha\left|\nu \wedge \widetilde{E}_{k}^{n}\right|^{2}+\frac{1}{\alpha}\left|\widetilde{H}_{k \tau}^{n}\right|^{2}\right) \mathrm{d} \Gamma \mathrm{d} t \leq\left\|\left(\widetilde{E}_{k}^{n}\left(T_{k}\right), \widetilde{H}_{k}^{n}\left(T_{k}\right)\right)\right\|_{\mathcal{H}}^{2} \\
& +\int_{T_{k}}^{t} \int_{\Gamma} \frac{1}{\alpha}\left|\nu \wedge \widetilde{P}_{k}^{n}\right|^{2} \mathrm{~d} \Gamma \mathrm{d} t
\end{aligned}
$$

valid for $T_{k} \leq t \leq T_{k+1}$ and $k=0, \ldots, K$. We write the first term on the right side of (6.13) as

$$
\left\|\left(\widetilde{E}_{k}^{n}\left(T_{k}\right), \widetilde{H}_{k}^{n}\left(T_{k}\right)\right)\right\|_{\mathcal{H}}^{2}-\left\|\left(\widetilde{E}_{k-1}^{n}\left(T_{k}\right), \widetilde{H}_{k-1}^{n}\left(T_{k}\right)\right)\right\|_{\mathcal{H}}^{2}+\left\|\left(\widetilde{E}_{k-1}^{n}\left(T_{k}\right), \widetilde{H}_{k-1}^{n}\left(T_{k}\right)\right)\right\|_{\mathcal{H}}^{2}
$$

and then use (6.12) again to estimate the last term as follows:

$$
\begin{aligned}
\left\|\left(\widetilde{E}_{k-1}^{n}\left(T_{k}\right), \widetilde{H}_{k-1}^{n}\left(T_{k}\right)\right)\right\|_{\mathcal{H}}^{2} \leq & -\int_{T_{k-1}}^{T_{k}} \int_{\Gamma}\left(\alpha\left|\nu \wedge \widetilde{E}_{k-1}^{n}\right|^{2}+\frac{1}{\alpha}\left|\widetilde{H}_{k-1, \tau}^{n}\right|^{2}\right) \mathrm{d} \Gamma \mathrm{d} t \\
& +\left\|\left(\widetilde{E}_{k-1}^{n}\left(T_{k-1}\right), \widetilde{H}_{k-1}^{n}\left(T_{k-1}\right)\right)\right\|_{\mathcal{H}}^{2}+\int_{T_{k-1}}^{T_{k}} \int_{\Gamma} \frac{1}{\alpha}\left|\nu \wedge \widetilde{P}_{k-1}^{n}\right|^{2} \mathrm{~d} \Gamma \mathrm{d} t .
\end{aligned}
$$

From (6.13-6.15) we then obtain

$$
\begin{aligned}
\left\|\left(\widetilde{E}_{k}^{n}(t), \widetilde{H}_{k}^{n}(t)\right)\right\|_{\mathcal{H}}^{2} \leq & +\int_{T_{k-1}}^{T_{k}} \int_{\Gamma}\left(\alpha\left|\nu \wedge \widetilde{E}_{k-1}^{n}\right|^{2}+\frac{1}{\alpha}\left|\widetilde{H}_{k-1, \tau}^{n}\right|^{2}\right) \mathrm{d} \Gamma \mathrm{d} t+\int_{T_{k}}^{t} \int_{\Gamma}\left(\alpha\left|\nu \wedge \widetilde{E}_{k}^{n}\right|^{2}+\frac{1}{\alpha}\left|\widetilde{H}_{k \tau}^{n}\right|^{2}\right) \mathrm{d} \Gamma \mathrm{d} t \\
\leq & \left(\left\|\left(\widetilde{E}_{k}^{n}\left(T_{k}\right), \widetilde{H}_{k}^{n}\left(T_{k}\right)\right)\right\|_{\mathcal{H}}^{2}-\left\|\left(\widetilde{E}_{k-1}^{n}\left(T_{k}\right), \widetilde{H}_{k-1}^{n}\left(T_{k}\right)\right)\right\|_{\mathcal{H}}^{2}\right)+\left\|\left(\widetilde{E}_{k-1}^{n}\left(T_{k-1}\right), \widetilde{H}_{k-1}^{n}\left(T_{k-1}\right)\right)\right\|_{\mathcal{H}}^{2} \\
& +\int_{T_{k-1}}^{T_{k}} \int_{\Gamma} \frac{1}{\alpha}\left|\nu \wedge \widetilde{P}_{k-1}^{n}\right|^{2} \mathrm{~d} \Gamma \mathrm{d} t+\int_{T_{k}}^{t} \int_{\Gamma} \frac{1}{\alpha}\left|\nu \wedge \widetilde{P}_{k}^{n}\right|^{2} \mathrm{~d} \Gamma \mathrm{d} t
\end{aligned}
$$


We now repeat the argument, using (6.14-6.16) with $k$ replaced by $k-1$, and so forth down to $k=0$. Since $\widetilde{E}_{0}^{n}(0)=\widetilde{H}_{0}^{n}(0)=0$ we end up with

$$
\begin{aligned}
\left\|\left(\widetilde{E}_{k}^{n}(t), \widetilde{H}_{k}^{n}(t)\right)\right\|_{\mathcal{H}}^{2} & +\sum_{m=0}^{k-1} \int_{T_{m}}^{T_{m+1}} \int_{\Gamma}\left(\alpha\left|\nu \wedge \widetilde{E}_{m}^{n}\right|^{2}+\frac{1}{\alpha}\left|\widetilde{H}_{m \tau}^{n}\right|^{2}\right) \mathrm{d} \Gamma \mathrm{d} t+\int_{T_{k}}^{t} \int_{\Gamma}\left(\alpha\left|\nu \wedge \widetilde{E}_{k}^{n}\right|^{2}+\frac{1}{\alpha}\left|\widetilde{H}_{k \tau}^{n}\right|^{2}\right) \mathrm{d} \Gamma \mathrm{d} t \\
\leq & \sum_{m=0}^{k-1}\left(\left\|\left(\widetilde{E}_{m+1}^{n}\left(T_{m+1}\right), \widetilde{H}_{m+1}^{n}\left(T_{m+1}\right)\right)\right\|_{\mathcal{H}}^{2}-\left\|\left(\widetilde{E}_{m}^{n}\left(T_{m+1}\right), \widetilde{H}_{m}^{n}\left(T_{m+1}\right)\right)\right\|_{\mathcal{H}}^{2}\right) \\
& +\sum_{m=0}^{k-1} \int_{T_{m}}^{T_{m+1}} \int_{\Gamma} \frac{1}{\alpha}\left|\nu \wedge \widetilde{P}_{m}^{n}\right|^{2} \mathrm{~d} \Gamma \mathrm{d} t+\int_{T_{k}}^{t} \int_{\Gamma} \frac{1}{\alpha}\left|\nu \wedge \widetilde{P}_{k}^{n}\right|^{2} \mathrm{~d} \Gamma \mathrm{d} t .
\end{aligned}
$$

This estimate is valid for $t \in I_{k}$ and $k=0, \ldots, K$.

By the Cauchy-Schwarz inequality we have

$$
\begin{aligned}
\sum_{m=0}^{k-1}\left(\left\|\left(\widetilde{E}_{m+1}^{n}\left(T_{m+1}\right), \widetilde{H}_{m+1}^{n}\left(T_{m+1}\right)\right)\right\|_{\mathcal{H}}^{2}-\left\|\left(\widetilde{E}_{m}^{n}\left(T_{m+1}\right), \widetilde{H}_{m}^{n}\left(T_{m+1}\right)\right)\right\|_{\mathcal{H}}^{2}\right) \\
\leq \sqrt{2}\left\{\sum_{m=0}^{k-1}\left\|\left(E_{m+1}^{n}\left(T_{m+1}\right), H_{m+1}^{n}\left(T_{m+1}\right)\right)-\left(E_{m}^{n}\left(T_{m+1}\right), H_{m}^{n}\left(T_{m+1}\right)\right)\right\|_{\mathcal{H}}^{2}\right\}^{1 / 2} \\
\times\left\{\sum_{m=0}^{k-1}\left(\left\|\left(\widetilde{E}_{m+1}^{n}\left(T_{m+1}\right), \widetilde{H}_{m+1}^{n}\left(T_{m+1}\right)\right)\right\|_{\mathcal{H}}^{2}+\left\|\left(\widetilde{E}_{m}^{n}\left(T_{m+1}\right), \widetilde{H}_{m}^{n}\left(T_{m+1}\right)\right)\right\|_{\mathcal{H}}^{2}\right)\right\}^{1 / 2} .
\end{aligned}
$$

It follows that

$$
\begin{aligned}
\max _{0 \leq k \leq K}\left\|\left(\widetilde{E}_{k}^{n}, \widetilde{H}_{k}^{n}\right)\right\|_{L^{\infty}\left(I_{k} ; \mathcal{H}\right)}^{2} \leq & \sqrt{2}\left\{\sum_{k=0}^{K-1}\left\|\left(E_{k+1}^{n}\left(T_{k+1}\right), H_{k+1}^{n}\left(T_{k+1}\right)\right)-\left(E_{k}^{n}\left(T_{k+1}\right), H_{k}^{n}\left(T_{k+1}\right)\right)\right\|_{\mathcal{H}}^{2}\right\}^{1 / 2} \\
& \times\left\{\sum_{k=0}^{K-1}\left(\left\|\left(\widetilde{E}_{k+1}^{n}\left(T_{k+1}\right), \widetilde{H}_{k+1}^{n}\left(T_{k+1}\right)\right)\right\|_{\mathcal{H}}^{2}+\left\|\left(\widetilde{E}_{k}^{n}\left(T_{k+1}\right), \widetilde{H}_{k}^{n}\left(T_{k+1}\right)\right)\right\|_{\mathcal{H}}^{2}\right)\right\}^{1 / 2} \\
& +\sum_{k=0}^{K} \int_{\Sigma_{k}} \frac{1}{\alpha}\left|\nu \wedge \widetilde{P}_{k}^{n}\right|^{2} \mathrm{~d} \Sigma
\end{aligned}
$$

and that

$$
\begin{aligned}
& \sum_{k=0}^{K} \int_{\Sigma_{k}}\left(\alpha\left|\nu \wedge \widetilde{E}_{k}^{n}\right|^{2}\right.\left.+\frac{1}{\alpha}\left|\widetilde{H}_{k \tau}^{n}\right|^{2}\right) \mathrm{d} \Sigma \\
& \leq \sqrt{2}\left\{\sum_{k=0}^{K-1}\left\|\left(E_{k+1}^{n}\left(T_{k+1}\right), H_{k+1}^{n}\left(T_{k+1}\right)\right)-\left(E_{k}^{n}\left(T_{k+1}\right), H_{k}^{n}\left(T_{k+1}\right)\right)\right\|_{\mathcal{H}}^{2}\right\}^{1 / 2} \\
& \times\left\{\sum_{k=0}^{K-1}\left(\left\|\left(\widetilde{E}_{k+1}^{n}\left(T_{k+1}\right), \widetilde{H}_{k+1}^{n}\left(T_{k+1}\right)\right)\right\|_{\mathcal{H}}^{2}+\left\|\left(\widetilde{E}_{k}^{n}\left(T_{k+1}\right), \widetilde{H}_{k}^{n}\left(T_{k+1}\right)\right)\right\|_{\mathcal{H}}^{2}\right)\right\}^{1 / 2} \\
&+\sum_{k=0}^{K} \int_{\Sigma_{k}} \frac{1}{\alpha}\left|\nu \wedge \widetilde{P}_{k}^{n}\right|^{2} \mathrm{~d} \Sigma .
\end{aligned}
$$


We estimate the solutions $\left(\widetilde{P}_{k}^{n}, \widetilde{Q}_{k}^{n}\right)$ in a similar manner, starting with the estimate

$$
\left\|\left(\widetilde{P}_{k}^{n}(t), \widetilde{Q}_{k}^{n}(t)\right)\right\|_{\mathcal{H}}^{2}+\int_{t}^{T_{k+1}} \int_{\Gamma}\left(\alpha\left|\nu \wedge \widetilde{P}_{k}^{n}\right|^{2}+\frac{1}{\alpha}\left|\widetilde{Q}_{k \tau}^{n}\right|^{2}\right) \mathrm{d} \Gamma \mathrm{d} t \leq\left\|\left(\widetilde{P}_{k}^{n}\left(T_{k+1}\right), \widetilde{Q}_{k}^{n}\left(T_{k+1}\right)\right)\right\|_{\mathcal{H}}^{2}, \quad t \in I_{k}
$$

The term on the right side is written

$$
\begin{aligned}
\left\|\left(\widetilde{P}_{k}^{n}\left(T_{k+1}\right), \widetilde{Q}_{k}^{n}\left(T_{k+1}\right)\right)\right\|_{\mathcal{H}}^{2} & -\left\|\left(\widetilde{P}_{k+1}^{n}\left(T_{k+1}\right), \widetilde{Q}_{k+1}^{n}\left(T_{k+1}\right)\right)\right\|_{\mathcal{H}}^{2} \\
& +\left\|\left(\widetilde{P}_{k+1}^{n}\left(T_{k+1}\right), \widetilde{Q}_{k+1}^{n}\left(T_{k+1}\right)\right)\right\|_{\mathcal{H}}^{2} \leq\left\|\left(\widetilde{P}_{k}^{n}\left(T_{k+1}\right), \widetilde{Q}_{k}^{n}\left(T_{k+1}\right)\right)\right\|_{\mathcal{H}}^{2} \\
& -\left\|\left(\widetilde{P}_{k+1}^{n}\left(T_{k+1}\right), \widetilde{Q}_{k+1}^{n}\left(T_{k+1}\right)\right)\right\|_{\mathcal{H}}^{2} \\
& +\left\|\left(\widetilde{P}_{k+1}^{n}\left(T_{k+2}\right), \widetilde{Q}_{k+1}^{n}\left(T_{k+2}\right)\right)\right\|_{\mathcal{H}}^{2} \\
& -\int_{T_{k+1}}^{T_{k+2}} \int_{\Gamma}\left(\alpha\left|\nu \wedge \widetilde{P}_{k+1}^{n}\right|^{2}+\frac{1}{\alpha}\left|\widetilde{Q}_{k+1, \tau}^{n}\right|^{2}\right) \mathrm{d} \Gamma \mathrm{d} t .
\end{aligned}
$$

From (6.21) and (6.22) we thus obtain

$$
\begin{aligned}
\left\|\left(\widetilde{P}_{k}^{n}(t), \widetilde{Q}_{k}^{n}(t)\right)\right\|_{\mathcal{H}}^{2} & +\int_{t}^{T_{k+1}} \int_{\Gamma}\left(\alpha\left|\nu \wedge \widetilde{P}_{k}^{n}\right|^{2}+\frac{1}{\alpha}\left|\widetilde{Q}_{k \tau}^{n}\right|^{2}\right) \mathrm{d} \Gamma \mathrm{d} t \\
& +\int_{T_{k+1}}^{T_{k+2}} \int_{\Gamma}\left(\alpha\left|\nu \wedge \widetilde{P}_{k+1}^{n}\right|^{2}+\frac{1}{\alpha}\left|\widetilde{Q}_{k+1, \tau}^{n}\right|^{2}\right) \mathrm{d} \Gamma \mathrm{d} t \leq\left\|\left(\widetilde{P}_{k}^{n}\left(T_{k+1}\right), \widetilde{Q}_{k}^{n}\left(T_{k+1}\right)\right)\right\|_{\mathcal{H}}^{2} \\
& -\left\|\left(\widetilde{P}_{k+1}^{n}\left(T_{k+1}\right), \widetilde{Q}_{k+1}^{n}\left(T_{k+1}\right)\right)\right\|_{\mathcal{H}}^{2} \\
& +\left\|\left(\widetilde{P}_{k+1}^{n}\left(T_{k+2}\right), \widetilde{Q}_{k+1}^{n}\left(T_{k+2}\right)\right)\right\|_{\mathcal{H}}^{2} .
\end{aligned}
$$

Iterating the argument leads finally to

$$
\begin{aligned}
\left\|\left(\widetilde{P}_{k}^{n}(t), \widetilde{Q}_{k}^{n}(t)\right)\right\|_{\mathcal{H}}^{2} & +\int_{t}^{T_{k+1}} \int_{\Gamma}\left(\alpha\left|\nu \wedge \widetilde{P}_{k}^{n}\right|^{2}+\frac{1}{\alpha}\left|\widetilde{Q}_{k \tau}^{n}\right|^{2}\right) \mathrm{d} \Gamma \mathrm{d} t \\
& +\sum_{m=k+1}^{K} \int_{T_{m}}^{T_{m+1}} \int_{\Gamma}\left(\alpha\left|\nu \wedge \widetilde{P}_{m}^{n}\right|^{2}+\frac{1}{\alpha}\left|\widetilde{Q}_{m \tau}^{n}\right|^{2}\right) \mathrm{d} \Gamma \mathrm{d} t \\
\leq & \sum_{m=k}^{K-1}\left(\left\|\left(\widetilde{P}_{m}^{n}\left(T_{m+1}\right), \widetilde{Q}_{m}^{n}\left(T_{m+1}\right)\right)\right\|_{\mathcal{H}}^{2}-\left\|\left(\widetilde{P}_{m+1}^{n}\left(T_{m+1}\right), \widetilde{Q}_{m+1}^{n}\left(T_{m+1}\right)\right)\right\|_{\mathcal{H}}^{2}\right) \\
& +\kappa^{2}\left\|\left(\widetilde{E}_{K}^{n}(T), \widetilde{H}_{K}^{n}(T)\right)\right\|_{\mathcal{H}}^{2}
\end{aligned}
$$

since $\left(\widetilde{P}_{K}^{n}(T), \widetilde{Q}_{K}^{n}(T)\right)=\kappa\left(\widetilde{E}_{K}^{n}(T), \widetilde{H}_{K}^{n}(T)\right)$. It follows that

$$
\begin{aligned}
\max _{0 \leq k \leq K}\left\|\left(\widetilde{P}_{k}^{n}, \widetilde{Q}_{k}^{n}\right)\right\|_{L^{\infty}\left(I_{k} ; \mathcal{H}\right)}^{2} \leq & \sqrt{2}\left\{\sum_{k=0}^{K-1}\left\|\left(P_{k}^{n}\left(T_{k+1}\right), Q_{k}^{n}\left(T_{k+1}\right)\right)-\left(P_{k+1}^{n}\left(T_{k+1}\right), Q_{k+1}^{n}\left(T_{k+1}\right)\right)\right\|_{\mathcal{H}}^{2}\right\}^{1 / 2} \\
& \times\left\{\sum_{k=0}^{K-1}\left(\left\|\left(\widetilde{P}_{k}^{n}\left(T_{k+1}\right), \widetilde{Q}_{k}^{n}\left(T_{k+1}\right)\right)\right\|_{\mathcal{H}}^{2}+\left\|\left(\widetilde{P}_{k+1}^{n}\left(T_{k+1}\right), \widetilde{Q}_{k+1}^{n}\left(T_{k+1}\right)\right)\right\|_{\mathcal{H}}^{2}\right)\right\}^{1 / 2} \\
& +\kappa^{2}\left\|\left(\widetilde{E}_{K}^{n}(T), \widetilde{H}_{K}^{n}(T)\right)\right\|_{\mathcal{H}}^{2}
\end{aligned}
$$


and that

$$
\begin{gathered}
\sum_{k=0}^{K} \int_{\Sigma_{k}}\left(\alpha\left|\nu \wedge \widetilde{P}_{k}^{n}\right|^{2}+\frac{1}{\alpha}\left|\widetilde{Q}_{k \tau}^{n}\right|^{2}\right) \mathrm{d} \Sigma \leq \sqrt{2}\left\{\sum_{k=0}^{K-1}\left\|\left(P_{k}^{n}\left(T_{k+1}\right), Q_{k}^{n}\left(T_{k+1}\right)\right)-\left(P_{k+1}^{n}\left(T_{k+1}\right), Q_{k+1}^{n}\left(T_{k+1}\right)\right)\right\|_{\mathcal{H}}^{2}\right\}^{1 / 2} \\
\times\left\{\sum_{k=0}^{K-1}\left(\left\|\left(\widetilde{P}_{k}^{n}\left(T_{k+1}\right), \widetilde{Q}_{k}^{n}\left(T_{k+1}\right)\right)\right\|_{\mathcal{H}}^{2}+\left\|\left(\widetilde{P}_{k+1}^{n}\left(T_{k+1}\right), \widetilde{Q}_{k+1}^{n}\left(T_{k+1}\right)\right)\right\|_{\mathcal{H}}^{2}\right)\right\}^{1 / 2} \\
+\kappa^{2}\left\|\left(\widetilde{E}_{K}^{n}(T), \widetilde{H}_{K}^{n}(T)\right)\right\|_{\mathcal{H}}^{2} \cdot \quad(6.25)
\end{gathered}
$$

We therefore obtain from $(6.19,6.20,6.24)$ and $(6.25)$

$$
\begin{aligned}
\mathrm{e}^{n} \leq & 2 \sqrt{2}\left\{\sum_{k=0}^{K-1}\left\|\left(E_{k+1}^{n}\left(T_{k+1}\right), H_{k+1}^{n}\left(T_{k+1}\right)\right)-\left(E_{k}^{n}\left(T_{k+1}\right), H_{k}^{n}\left(T_{k+1}\right)\right)\right\|_{\mathcal{H}}^{2}\right\}^{1 / 2} \\
& \times\left\{\sum_{k=0}^{K-1}\left(\left\|\left(\widetilde{E}_{k+1}^{n}\left(T_{k+1}\right), \widetilde{H}_{k+1}^{n}\left(T_{k+1}\right)\right)\right\|_{\mathcal{H}}^{2}+\left\|\left(\widetilde{E}_{k}^{n}\left(T_{k+1}\right), \widetilde{H}_{k}^{n}\left(T_{k+1}\right)\right)\right\|_{\mathcal{H}}^{2}\right)\right\}^{1 / 2} \\
& +2 \sqrt{2}\left\{\sum_{k=0}^{K-1}\left\|\left(P_{k}^{n}\left(T_{k+1}\right), Q_{k}^{n}\left(T_{k+1}\right)\right)-\left(P_{k+1}^{n}\left(T_{k+1}\right), Q_{k+1}^{n}\left(T_{k+1}\right)\right)\right\|_{\mathcal{H}}^{2}\right\}^{1 / 2} \\
& \times\left\{\sum_{k=0}^{K-1}\left(\left\|\left(\widetilde{P}_{k}^{n}\left(T_{k+1}\right), \widetilde{Q}_{k}^{n}\left(T_{k+1}\right)\right)\right\|_{\mathcal{H}}^{2}+\left\|\left(\widetilde{P}_{k+1}^{n}\left(T_{k+1}\right), \widetilde{Q}_{k+1}^{n}\left(T_{k+1}\right)\right)\right\|_{\mathcal{H}}^{2}\right)\right\}^{1 / 2} \\
& +2 \sum_{k=0}^{K} \int_{\Sigma_{k}} \frac{1}{\alpha}\left|\nu \wedge \widetilde{P}_{k}^{n}\right|^{2} \mathrm{~d} \Sigma+2 \kappa^{2}\left\|\left(\widetilde{E}_{K}^{n}(T), \widetilde{H}_{K}^{n}(T)\right)\right\|_{\mathcal{H}}^{2} .
\end{aligned}
$$

By applying the Cauchy-Schwarz ineqality once again and recalling the definitions of $\mathcal{E}_{\beta}^{n}$ and $\mathcal{E}_{k, k+1}^{n}\left(T_{k+1}\right)$ we obtain

$$
\mathrm{e}^{n} \leq 2 \sqrt{2} \max (1,1 / \beta) \sqrt{\mathcal{E}_{\beta}^{n}}\left\{\sum_{k=0}^{K-1} \mathcal{E}_{k, k+1}^{n}\left(T_{k+1}\right)\right\}^{1 / 2}+2 \sum_{k=0}^{K} \int_{\Sigma_{k}} \frac{1}{\alpha}\left|\nu \wedge \widetilde{P}_{k}^{n}\right|^{2} \mathrm{~d} \Sigma+2 \kappa^{2}\left\|\left(\widetilde{E}_{K}^{n}(T), \widetilde{H}_{K}^{n}(T)\right)\right\|_{\mathcal{H}}^{2} .
$$

From the definition of $\mathcal{F}_{\beta}^{n}$ we obtain

$$
2 \sum_{k=0}^{K} \int_{\Sigma_{k}} \frac{1}{\alpha}\left|\nu \wedge \widetilde{P}_{k}^{n}\right|^{2} \mathrm{~d} \Sigma+2 \kappa^{2}\left\|\left(\widetilde{E}_{K}^{n}(T), \widetilde{H}_{K}^{n}(T)\right)\right\|_{\mathcal{H}}^{2} \leq \frac{1}{\beta} \max \left(\left\|\alpha^{-1}\right\|_{L^{\infty}(\Gamma)}, \kappa\right) \mathcal{F}_{\beta}^{n} .
$$

Lemma 6.2 follows from (6.27) and (6.28).

Proof of Lemma 6.3. We start with our fundamental recursion which we now write as

$$
\mathcal{F}_{\beta}^{n+1}+(1-2 \epsilon) \mathcal{F}_{\beta}^{n} \leq \mathcal{E}_{\beta}^{n}-\mathcal{E}_{\beta}^{n+1}
$$


We proceed to estimate

$$
\mathcal{E}_{\beta}^{n}-\mathcal{E}_{\beta}^{n+1}=\sum_{k=0}^{K-1}\left\{\left[\mathcal{E}_{k, \beta}^{n}\left(T_{k+1}\right)-\mathcal{E}_{k+1, \beta}^{n+1}\left(T_{k+1}\right)\right]+\left[\mathcal{E}_{k+1, \beta}^{n}\left(T_{k+1}\right)-\mathcal{E}_{k, \beta}^{n+1}\left(T_{k+1}\right)\right]\right\}
$$

We have

$$
\begin{aligned}
\mathcal{E}_{k, \beta}^{n}\left(T_{k+1}\right)-\mathcal{E}_{k+1, \beta}^{n+1}\left(T_{k+1}\right)= & \beta^{2}\left(\left\|\left(\widetilde{E}_{k}^{n}\left(T_{k+1}\right), \widetilde{H}_{k}^{n}\left(T_{k+1}\right)\right)\right\|_{\mathcal{H}}-\left\|\left(\widetilde{E}_{k+1}^{n+1}\left(T_{k+1}\right), \widetilde{H}_{k+1}^{n+1}\left(T_{k+1}\right)\right)\right\|_{\mathcal{H}}\right) \\
& \times\left(\left\|\left(\widetilde{E}_{k}^{n}\left(T_{k+1}\right), \widetilde{H}_{k}^{n}\left(T_{k+1}\right)\right)\right\|_{\mathcal{H}}+\left\|\left(\widetilde{E}_{k+1}^{n+1}\left(T_{k+1}\right), \widetilde{H}_{k+1}^{n+1}\left(T_{k+1}\right)\right)\right\|_{\mathcal{H}}\right) \\
& +\left(\left\|\left(\widetilde{P}_{k}^{n}\left(T_{k+1}\right), \widetilde{Q}_{k}^{n}\left(T_{k+1}\right)\right)\right\|_{\mathcal{H}}-\left\|\left(\widetilde{P}_{k+1}^{n+1}\left(T_{k+1}\right), \widetilde{Q}_{k+1}^{n+1}\left(T_{k+1}\right)\right)\right\|_{\mathcal{H}}\right) \\
& \times\left(\left\|\left(\widetilde{P}_{k}^{n}\left(T_{k+1}\right), \widetilde{Q}_{k}^{n}\left(T_{k+1}\right)\right)\right\|_{\mathcal{H}}+\left\|\left(\widetilde{P}_{k+1}^{n+1}\left(T_{k+1}\right), \widetilde{Q}_{k+1}^{n+1}\left(T_{k+1}\right)\right)\right\|_{\mathcal{H}}\right) .
\end{aligned}
$$

Therefore by the Cauchy-Schwarz inequality we obtain

$$
\begin{array}{r}
\sum_{k=0}^{K-1}\left(\mathcal{E}_{k, \beta}^{n}\left(T_{k+1}\right)-\mathcal{E}_{k+1, \beta}^{n+1}\left(T_{k+1}\right)\right) \leq \sqrt{2}\left\{\sum _ { k = 0 } ^ { K - 1 } \left[\beta^{2}\left\|\left(E_{k}^{n}\left(T_{k+1}\right)-E_{k+1}^{n+1}\left(T_{k+1}\right), H_{k}^{n}\left(T_{k+1}\right)-H_{k+1}^{n+1}\left(T_{k+1}\right)\right)\right\|_{\mathcal{H}}^{2}\right.\right. \\
\left.\left.\quad+\left\|\left(P_{k}^{n}\left(T_{k+1}\right)-P_{k+1}^{n+1}\left(T_{k+1}\right), Q_{k}^{n}\left(T_{k+1}\right)-Q_{k+1}^{n+1}\left(T_{k+1}\right)\right)\right\|_{\mathcal{H}}^{2}\right]\right\}^{1 / 2} \\
\times\left\{\sum _ { k = 0 } ^ { K - 1 } \left[\beta^{2}\left(\left\|\left(\widetilde{E}_{k}^{n}\left(T_{k+1}\right), \widetilde{H}_{k}^{n}\left(T_{k+1}\right)\right)\right\|_{\mathcal{H}}^{2}+\left\|\left(\widetilde{E}_{k+1}^{n+1}\left(T_{k+1}\right), \widetilde{H}_{k+1}^{n+1}\left(T_{k+1}\right)\right)\right\|_{\mathcal{H}}^{2}\right)\right.\right. \\
\left.\left.+\left\|\left(\widetilde{P}_{k}^{n}\left(T_{k+1}\right), \widetilde{Q}_{k}^{n}\left(T_{k+1}\right)\right)\right\|_{\mathcal{H}}^{2}+\left\|\left(\widetilde{P}_{k+1}^{n+1}\left(T_{k+1}\right), \widetilde{Q}_{k+1}^{n+1}\left(T_{k+1}\right)\right)\right\|_{\mathcal{H}}^{2}\right]\right\}^{1 / 2} \\
\leq \sqrt{2} \max (1, \beta)\left\{\sum_{k=0}^{K-1} \mathcal{E}_{k, k+1}^{n, n+1}\left(T_{k+1}\right)\right\}^{1 / 2}\left\{\sum_{k=0}^{K-1} B_{k, k+1}^{n, n+1}\left(T_{k+1}\right)\right\}^{1 / 2}
\end{array}
$$

where

$$
\begin{aligned}
B_{k, k+1}^{n, n+1}\left(T_{k+1}\right)= & \beta^{2}\left(\left\|\left(\widetilde{E}_{k}^{n}\left(T_{k+1}\right), \widetilde{H}_{k}^{n}\left(T_{k+1}\right)\right)\right\|_{\mathcal{H}}^{2}+\left\|\left(\widetilde{E}_{k+1}^{n+1}\left(T_{k+1}\right), \widetilde{H}_{k+1}^{n+1}\left(T_{k+1}\right)\right)\right\|_{\mathcal{H}}^{2}\right) \\
& +\left\|\left(\widetilde{P}_{k}^{n}\left(T_{k+1}\right), \widetilde{Q}_{k}^{n}\left(T_{k+1}\right)\right)\right\|_{\mathcal{H}}^{2}+\left\|\left(\widetilde{P}_{k+1}^{n+1}\left(T_{k+1}\right), \widetilde{Q}_{k+1}^{n+1}\left(T_{k+1}\right)\right)\right\|_{\mathcal{H}}^{2} .
\end{aligned}
$$

Similarly

$$
\sum_{k=0}^{K-1}\left(\mathcal{E}_{k+1, \beta}^{n}\left(T_{k+1}\right)-\mathcal{E}_{k, \beta}^{n+1}\left(T_{k+1}\right)\right) \leq \sqrt{2} \max (1, \beta)\left\{\sum_{k=0}^{K-1} \mathcal{E}_{k+1, k}^{n, n+1}\left(T_{k+1}\right)\right\}^{1 / 2}\left\{\sum_{k=0}^{K-1} B_{k+1, k}^{n, n+1}\left(T_{k+1}\right)\right\}^{1 / 2}
$$

It follows from $(6.30-6.32)$ that

$$
\begin{aligned}
\mathcal{E}_{\beta}^{n}-\mathcal{E}_{\beta}^{n+1} \leq & \sqrt{2} \max (1, \beta)\left\{\sum_{k=0}^{K-1}\left(B_{k, k+1}^{n, n+1}\left(T_{k+1}\right)+B_{k+1, k}^{n, n+1}\left(T_{k+1}\right)\right)\right\}^{1 / 2} \\
& \times\left\{\sum_{k=0}^{K-1}\left(\mathcal{E}_{k, k+1}^{n, n+1}\left(T_{k+1}\right)+\mathcal{E}_{k+1, k}^{n, n+1}\left(T_{k+1}\right)\right)\right\}^{1 / 2} .
\end{aligned}
$$


The proof is completed by noting that

$$
\sum_{k=0}^{K-1}\left(B_{k, k+1}^{n, n+1}\left(T_{k+1}\right)+B_{k+1, k}^{n, n+1}\left(T_{k+1}\right)\right)=\mathcal{E}_{\beta}^{n+1}+\mathcal{E}_{\beta}^{n} .
$$

Proof of Lemma 6.4. From $(6.17,6.23)$ and $(6.28)$ we have

$$
\begin{aligned}
\mathrm{e}_{k}^{n+1}(t)+\mathrm{e}_{k}^{n}(t) \leq & \sum_{m=0}^{k-1}\left(\left\|\left(\widetilde{E}_{m+1}^{n+1}\left(T_{m+1}\right), \widetilde{H}_{m+1}^{n+1}\left(T_{m+1}\right)\right)\right\|_{\mathcal{H}}^{2}-\left\|\left(\widetilde{E}_{m}^{n}\left(T_{m+1}\right), \widetilde{H}_{m}^{n}\left(T_{m+1}\right)\right)\right\|_{\mathcal{H}}^{2}\right. \\
& \left.+\left\|\left(\widetilde{E}_{m+1}^{n}\left(T_{m+1}\right), \widetilde{H}_{m+1}^{n}\left(T_{m+1}\right)\right)\right\|_{\mathcal{H}}^{2}-\left\|\left(\widetilde{E}_{m}^{n+1}\left(T_{m+1}\right), \widetilde{H}_{m}^{n+1}\left(T_{m+1}\right)\right)\right\|_{\mathcal{H}}^{2}\right) \\
& +\sum_{m=k}^{K-1}\left(\left\|\left(\widetilde{P}_{m}^{n+1}\left(T_{m+1}\right), \widetilde{Q}_{m}^{n+1}\left(T_{m+1}\right)\right)\right\|_{\mathcal{H}}^{2}-\left\|\left(\widetilde{P}_{m+1}^{n}\left(T_{m+1}\right), \widetilde{Q}_{m+1}^{n}\left(T_{m+1}\right)\right)\right\|_{\mathcal{H}}^{2}\right. \\
& \left.+\left\|\left(\widetilde{P}_{m}^{n}\left(T_{m+1}\right), \widetilde{Q}_{m}^{n}\left(T_{m+1}\right)\right)\right\|_{\mathcal{H}}^{2}-\left\|\left(\widetilde{P}_{m+1}^{n+1}\left(T_{m+1}\right), \widetilde{Q}_{m+1}^{n+1}\left(T_{m+1}\right)\right)\right\|_{\mathcal{H}}^{2}\right) \\
& +\frac{1}{\beta} \max \left(\left\|\alpha^{-1}\right\|_{L^{\infty}(\Gamma)}, \kappa\right)\left(\mathcal{F}^{n+1}+\mathcal{F}_{\beta}^{n}\right) .
\end{aligned}
$$

The sums on the right are estimated as in (6.18), resulting in

$$
\begin{aligned}
\max _{0 \leq k \leq K}\left\|e_{k}^{n+1}+e_{k}^{n}\right\|_{L^{\infty}(0, T)} \leq & \sqrt{2}\left\{\sum _ { k = 0 } ^ { K - 1 } \left(\left\|\left(E_{k+1}^{n+1}\left(T_{k+1}\right), H_{k+1}^{n+1}\left(T_{k+1}\right)\right)-\left(E_{k}^{n}\left(T_{k+1}\right), H_{k}^{n}\left(T_{k+1}\right)\right)\right\|_{\mathcal{H}}^{2}\right.\right. \\
& \left.\left.+\left\|\left(E_{k+1}^{n}\left(T_{k+1}\right), H_{k+1}^{n}\left(T_{k+1}\right)\right)-\left(E_{k}^{n+1}\left(T_{k+1}\right), H_{k}^{n+1}\left(T_{k+1}\right)\right)\right\|_{\mathcal{H}}^{2}\right)\right\}^{1 / 2} \\
& \times\left\{\sum _ { k = 0 } ^ { K - 1 } \left(\left\|\left(\widetilde{E}_{k+1}^{n+1}\left(T_{k+1}\right), \widetilde{H}_{k+1}^{n+1}\left(T_{k+1}\right)\right)\right\|_{\mathcal{H}}^{2}+\left\|\left(\widetilde{E}_{k}^{n}\left(T_{k+1}\right), \widetilde{H}_{k}^{n}\left(T_{k+1}\right)\right)\right\|_{\mathcal{H}}^{2}\right.\right. \\
& \left.\left.+\left\|\left(\widetilde{E}_{k+1}^{n}\left(T_{k+1}\right), \widetilde{H}_{k+1}^{n}\left(T_{k+1}\right)\right)\right\|_{\mathcal{H}}^{2}+\left\|\left(\widetilde{E}_{k}^{n+1}\left(T_{k+1}\right), \widetilde{H}_{k}^{n+1}\left(T_{k+1}\right)\right)\right\|_{\mathcal{H}}^{2}\right)\right\}^{1 / 2} \\
& +\sqrt{2}\left\{\sum _ { k = 0 } ^ { K - 1 } \left(\left\|\left(P_{k}^{n+1}\left(T_{k+1}\right), Q_{k}^{n+1}\left(T_{k+1}\right)\right)-\left(P_{k+1}^{n}\left(T_{k+1}\right), Q_{k+1}^{n}\left(T_{k+1}\right)\right)\right\|_{\mathcal{H}}^{2}\right.\right. \\
& \left.\left.+\left\|\left(P_{k}^{n}\left(T_{k+1}\right), Q_{k}^{n}\left(T_{k+1}\right)\right)-\left(P_{k+1}^{n+1}\left(T_{k+1}\right), Q_{k+1}^{n+1}\left(T_{k+1}\right)\right)\right\|_{\mathcal{H}}^{2}\right)\right\}^{1 / 2} \\
& \times\left\{\sum_{k=0}^{K-1}\left(\| \widetilde{P}_{k}^{n+1}\left(T_{k+1}\right), \widetilde{Q}_{k}^{n+1}\left(T_{k+1}\right)\right)\left\|_{\mathcal{H}}^{2}+\right\|\left(\widetilde{P}_{k+1}^{n}\left(T_{k+1}\right), \widetilde{Q}_{k+1}^{n}\left(T_{k+1}\right)\right) \|_{\mathcal{H}}^{2}\right. \\
& \left.\left.+\| \widetilde{P}_{k}^{n}\left(T_{k+1}\right), \widetilde{Q}_{k}^{n}\left(T_{k+1}\right)\right)\left\|_{\mathcal{H}}^{2}+\right\|\left(\widetilde{P}_{k+1}^{n+1}\left(T_{k+1}\right), \widetilde{Q}_{k+1}^{n+1}\left(T_{k+1}\right)\right) \|_{\mathcal{H}}^{2}\right\}^{1 / 2} \\
& +\frac{1}{\beta} \max \left(\left\|\alpha^{-1}\right\|_{L^{\infty}(\Gamma)}, \kappa\right)\left(\mathcal{F}^{n+1}+\mathcal{F}_{\beta}^{n}\right) .
\end{aligned}
$$

After one more application of the Cauchy-Schwarz inequality we obtain the conclusion of Lemma 6.4. 
Proof of Corollary 6.1. One has the rough estimate

$$
\mathcal{E}_{\beta}^{n}+\mathcal{F}_{\beta}^{n} \leq \mathcal{E}_{\beta}^{1}+\mathcal{F}_{\beta}^{1}
$$

We proceed to estimate $\mathcal{E}_{\beta}^{1}+\mathcal{F}_{\beta}^{1}$. From (5.21) and (5.10) we have

$$
\mathcal{E}_{\beta}^{1}+\mathcal{F}_{\beta}^{1}=\sum_{k=0}^{K-1}\left(\left\|\left(\tilde{\mu}_{k, k+1}^{0}, \tilde{\eta}_{k, k+1}^{0}\right)\right\|_{\mathcal{H}}^{2}+\left\|\left(\tilde{\mu}_{k+1, k}^{0}, \tilde{\eta}_{k+1, k}^{0}\right)\right\|_{\mathcal{H}}^{2}\right) .
$$

By (5.7) the right side of (6.36) is bounded above by

$$
\begin{aligned}
2 \sum_{k=0}^{K-1}\left(\left\|\left(\mu_{k, k+1}^{0}, \eta_{k, k+1}^{0}\right)\right\|_{\mathcal{H}}^{2}\right. & \left.+\left\|\left(\mu_{k+1, k}^{0}, \eta_{k+1, k}^{0}\right)\right\|_{\mathcal{H}}^{2}\right)+2 \sum_{k=0}^{K-1}\left(\beta^{2}\left\|\left(E\left(T_{k+1}\right), H\left(T_{k+1}\right)\right)\right\|_{\mathcal{H}}^{2}\right. \\
& \left.+\left\|\left(P\left(T_{k+1}\right), Q\left(T_{k+1}\right)\right)\right\|_{\mathcal{H}}^{2}\right) .
\end{aligned}
$$

We have the following a priori estimate for the solution of (2.1), which can be proved by standard multiplier methods (see [11])

$$
(1-\delta)\|(E, H)\|_{L^{\infty}(0, T ; \mathcal{H})}^{2} \leq\|(\phi, \psi)\|_{\mathcal{H}}^{2}+\int_{\Sigma} \frac{1}{\alpha}|J|^{2} \mathrm{~d} \Sigma+\frac{1}{\delta}\left\|\left(\varepsilon^{-1} F, \mu^{-1} G\right)\right\|_{L^{1}(0, T ; \mathcal{H})}^{2}, \quad 0<\delta<1 .
$$

Thus for every $k=0, \ldots, K-1$ we have

$$
\left\|\left(E\left(T_{k+1}\right), H\left(T_{k+1}\right)\right)\right\|_{\mathcal{H}}^{2} \leq 2\|(\phi, \psi)\|_{\mathcal{H}}^{2}+2 \int_{\Sigma} \frac{1}{\alpha}|\nu \wedge P|^{2} \mathrm{~d} \Sigma+4\left\|\left(\varepsilon^{-1} F, \mu^{-1} G\right)\right\|_{L^{1}(0, T ; \mathcal{H})}^{2} .
$$

In addition, from (6.12),

$$
\left\|\left(P\left(T_{k+1}\right), Q\left(T_{k+1}\right)\right)\right\|_{\mathcal{H}}^{2} \leq z^{2}\left\|\left(E(T)-E_{T}, H(T)-H_{T}\right)\right\|_{\mathcal{H}}^{2} .
$$

Thus the right side of (6.37) is bounded above by

$$
\begin{aligned}
& 2 \sum_{k=0}^{K-1}\left(\left\|\left(\mu_{k, k+1}^{0}, \eta_{k, k+1}^{0}\right)\right\|_{\mathcal{H}}^{2}+\right.\left.\left\|\left(\mu_{k+1, k}^{0}, \eta_{k+1, k}^{0}\right)\right\|_{\mathcal{H}}^{2}\right) \\
&+2 K\left\{2 \beta^{2}\|(\phi, \psi)\|_{\mathcal{H}}^{2}+4 \beta^{2}\left\|\left(\varepsilon^{-1} F, \mu^{-1} G\right)\right\|_{L^{1}(0, T ; \mathcal{H})}^{2}\right. \\
&\left.+\max \left(2 \beta^{2}\left\|\alpha^{-1}\right\|_{L^{\infty}(\Gamma)}, z\right)\left(\int_{\Sigma}|\nu \wedge P|^{2} \mathrm{~d} \Sigma+z\left\|\left(E(T)-E_{T}, H(T)-H_{T}\right)\right\|_{\mathcal{H}}^{2}\right)\right\}
\end{aligned}
$$

Since $\mathcal{J}\left(\left.\nu \wedge P\right|_{\Sigma}\right) \leq \mathcal{J}(0)$, we have

$$
\int_{\Sigma}|\nu \wedge P|^{2} \mathrm{~d} \Sigma+z\left\|\left(E(T)-E_{T}, H(T)-H_{T}\right)\right\|_{\mathcal{H}}^{2} \leq z\left\|\left(\hat{E}(T)-E_{T}, \hat{H}(T)-H_{T}\right)\right\|_{\mathcal{H}}^{2}
$$

where $(\hat{E}, \hat{H})$ is the solution of $(2.1)$ corresponding to $J=0$. Therefore

$$
\|(\hat{E}(T), \hat{H}(T))\|_{\mathcal{H}}^{2} \leq 2\|(\phi, \psi)\|_{\mathcal{H}}^{2}+4\left\|\left(\varepsilon^{-1} F, \mu^{-1} G\right)\right\|_{L^{1}(0, T ; \mathcal{H})}^{2},
$$


hence

$$
\begin{aligned}
\int_{\Sigma}|\nu \wedge P|^{2} \mathrm{~d} \Sigma+z\left\|\left(E(T)-E_{T}, H(T)-H_{T}\right)\right\|_{\mathcal{H}}^{2} \leq & 2 z\left(2\|(\phi, \psi)\|_{\mathcal{H}}^{2}+4\left\|\left(\varepsilon^{-1} F, \mu^{-1} G\right)\right\|_{L^{1}(0, T ; \mathcal{H})}^{2}\right. \\
& \left.+\left\|\left(E_{T}, H_{T}\right)\right\|_{\mathcal{H}}^{2}\right) .
\end{aligned}
$$

It follows from (6.36-6.40) that

$$
\left(\mathcal{E}_{\beta}^{1}+\mathcal{F}_{\beta}^{1}\right) \leq\left(C_{\beta}\right)^{2}
$$

where

$$
\begin{aligned}
C_{\beta}= & \sqrt{2}\left\{\sum_{k=0}^{K-1}\left(\left\|\left(\mu_{k, k+1}^{0}, \eta_{k, k+1}^{0}\right)\right\|_{\mathcal{H}}^{2}+\left\|\left(\mu_{k+1, k}^{0}, \eta_{k+1, k}^{0}\right)\right\|_{\mathcal{H}}^{2}\right)+2\left(K \beta^{2}+z \max \left(\beta^{2}\left\|\alpha^{-1}\right\|_{L^{\infty}(\Gamma)}, z\right)\right)\left(\|(\phi, \psi)\|_{\mathcal{H}}^{2}\right.\right. \\
& \left.\left.\left.+2\left\|\left(\varepsilon^{-1} F, \mu^{-1} G\right)\right\|_{L^{1}(0, T ; \mathcal{H})}^{2}\right)+z \max \left(\beta^{2}\left\|\alpha^{-1}\right\|_{L^{\infty}(\Gamma)}, z\right)\left\|\left(E_{T}, H_{T}\right)\right\|_{\mathcal{H}}^{2}\right)\right\}^{1 / 2}
\end{aligned}
$$

We therefore obtain (6.4) with

$$
C=C_{\beta} \sqrt{2} \max (1,1 / \beta)\left(2+\max \left(\left\|\alpha^{-1}\right\|_{L^{\infty}(\Gamma)}, z\right)\right) .
$$

Proof of Corollary 6.2. Since $\sqrt{\mathcal{E}_{\beta}^{n+1}+\mathcal{E}_{\beta}^{n}} \leq \sqrt{2} \sqrt{\mathcal{E}_{\beta}^{1}+\mathcal{F}_{\beta}^{1}}$, Corollary 6.2 follows immediately from (6.41), where

$$
C_{\epsilon}:=2 C_{\beta} \max (1,1 / \beta)\left(2+(1-2 \epsilon)^{-1} \max \left(\left\|\alpha^{-1}\right\|_{L^{\infty}(\Gamma)}, z\right)\right),
$$

where $C_{\beta}$ is given by $(6.42)$.

\section{REFERENCES}

[1] A. Alonso and A. Valli, An optimal domain decomposition preconditioner for low-frequency time-harmonic Maxwell equations. Math. Comp. 68 (1999) 607-631.

[2] M. Belishev and A. Glasman, Boundary control of the Maxwell dynamical system: Lack of controllability by topological reason. ESAIM: COCV 5 (2000) 207-218.

[3] J.-D. Benamou, Décomposition de domaine pour le contrôle de systèmes gouvernés par des équations d'évolution. $C$. $R$. Acad. Sci Paris Sér. I Math. 324 (1997) 1065-1070.

[4] J.-D. Benamou, Domain decomposition, optimal control of systems governed by partial differential equations and synthesis of feedback laws. J. Opt. Theory Appl. 102 (1999) 15-36.

[5] J.-D. Benamou and B. Desprès, A domain decomposition method for the Helmholtz equation and related optimal control problems. J. Comp. Phys. 136 (1997) 68-82.

[6] M. Gander, L. Halpern and F. Nataf, Optimal Schwarz waveform relaxation for the one dimensional wave equation. École Polytechnique, Palaiseau, Rep. 469 (2001).

[7] M. Heinkenschloss, Time domain decomposition iterative methods for the solution of distributed linear quadratic optimal control problems (submitted).

[8] J.E. Lagnese, A nonoverlapping domain decomposition for optimal boundary control of the dynamic Maxwell system, in Control of Nonlinear Distributed Parameter Systems, edited by G. Chen, I. Lasiecka and J. Zhou. Marcel Dekker (2001) 157-176.

[9] J.E. Lagnese, Exact boundary controllability of Maxwell's equation in a general region. SIAM J. Control Optim. 27 (1989) 374-388. 
[10] J.E. Lagnese and G. Leugering, Dynamic domain decomposition in approximate and exact boundary control problems of transmission for the wave equation. SIAM J. Control Optim. 38/2 (2000) 503-537.

[11] J.E. Lagnese, A singular perturbation problem in exact controllability of the Maxwell system. ESAIM: COCV 6 (2001) 275-290.

[12] J.-L. Lions, Virtual and effective control for distributed parameter systems and decomposition of everything. J. Anal. Math. 80 (2000) 257-297.

[13] J.-L. Lions, Decomposition of energy space and virtual control for parabolic systems, in 12th Int. Conf. on Domain Decomposition Methods, edited by T. Chan, T. Kako, H. Kawarada and O. Pironneau (2001) 41-53.

[14] J.-L. Lions and O. Pironneau, Domain decomposition methods for C.A.D. C. R. Acad. Sci. Paris 328 (1999) 73-80.

[15] Kim Dang Phung, Contrôle et stabilisation d'ondes électromagnétiques. ESAIM: COCV 5 (2000) 87-137.

[16] J.E. Santos, Global and domain decomposed mixed methods for the solution of Maxwell's equations with applications to magneotellurics. Num. Meth. for PDEs 14/4 (2000) 407-438.

[17] H. Schaefer, Über die Methode sukzessiver Approximationen. Jber Deutsch. Math.-Verein 59 (1957) 131-140. 